\title{
$\begin{array}{lllllll}\mathbf{R} & \mathbf{E} & \mathbf{V} & \mathbf{I} & \mathbf{S} & \mathbf{T} & \mathbf{A}\end{array}$ \\ ECONOMICA
}

LA PLATA

\section{Gasto público en salud: Envejecimiento, tecnología, crecimiento y no observables}

\section{Public spending on health: Aging, technology, growth and unobservables}

\author{
Facundo Luis Crosta \\ Rocío Navaridas \\ Lara Robla Vilá
}

\begin{abstract}
RESUMEN
Este documento establece que un modelo sencillo con efectos idiosincráticos por país, basado en ingresos, envejecimiento y una tendencia creciente de costos, posee gran capacidad explicativa del gasto público real por persona. Se extiende el ejercicio a tres niveles regionales: el mundo y regiones, América Latina y Argentina, durante 2000-2015. En este último caso, puede concluirse que el envejecimiento se asocia a los gastos incurridos previo al fallecimiento y una baja relevancia de "Enfermedad de costos" de Baumol.
\end{abstract}

Palabras claves: Argentina, Gasto Público en Salud, Determinantes, Ingreso, Envejecimiento, Tecnología, Enfermedad de costos.

\begin{abstract}
This document establishes that a simple model that captures the idiosyncratic effects of each country, based on income, aging and an increasing trend in costs, has great explanatory capacity of real public spending per person. The exercise is extended, to three regional levels: the world and its regions, Latin America and Argentina, for 2000-2015. For the latter case we can conclude that aging is associated with expenses incurred prior to death and a low significance of Baumol's Cost Disease.
\end{abstract}

Keywords: Argentina, Health Public Expenditure, determinants, Income, Aging, Technology, Cost Disease. 


\section{INTRODUCCIÓN}

La preocupación por la evolución creciente del gasto agregado en salud a nivel mundial surge tanto de cuestiones sanitarias y sociales como económicas. Entre las primeras se encuentran el logro de la cobertura universal $^{1}$ mientras que en las segundas predominan las presiones sobre el financiamiento. Esta tensión entre ambas fuerzas se resuelve en cada sistema de salud de manera diferente, pero la tendencia generalizada a sustituir gasto de bolsillo de los hogares con gasto público iniciada en la crisis de $2008^{2}$ motiva preguntarse sobre cuál será la evolución futura del gasto en salud, para lo cual resulta elemental conocer en profundidad sus determinantes. Es en este sentido, el presente trabajo apunta a estudiar los determinantes del gasto público en salud.

Los primeros estudios sobre determinantes del nivel de gasto agregado ${ }^{3}$ se centran en los niveles de ingreso y el estudio de su elasticidad, aspectos sobre los cuales todavía se sigue investigando con la intención de dilucidar si el bien (gasto en) salud es necesario o de lujo ${ }^{4}$. En este sentido, el análisis regional con relación a las condiciones de desarrollo siempre ha estado presente debido a que los cambios en el ingreso se asocian tanto a modificaciones en las expectativas de tratamientos como en la cantidad disponible de los mismos.

El envejecimiento poblacional, en especial de los países que aún no culminan la transición demográfica, afecta a los sistemas de salud por los cambios que induce en la demanda (cantidad, calidad y tipo) de servicios y, por lo tanto, en la demanda de recursos ${ }^{5}$. Cálculos relativamente sencillos permiten concluir que la reducción de la mortalidad infantil a la par de los aumentos en la esperanza de vida, conllevan a un aumento en el gasto promedio por persona, el cual puede ser aún mayor en la medida en que los cambios tecnológicos asociados a este nuevo perfil demográfico impliquen mayores $\operatorname{costos}^{6}$. La cuestión es si este resultado también es cierto para regiones con menor desarrollo, con menor capacidad fiscal y menores posibilidades institucionales, pero que se desenvuelven en un mundo más globalizado que podría compensar estas debilidades en la aplicación de procedimientos y técnicas de vanguardia con la mayor fluidez del conocimiento ${ }^{7}$.

Estos análisis deben ser ponderados por algunos hechos cuyos efectos todavía no están consolidados con relación al perfil epidemiológico y los gastos agregados en salud. Uno, es la existencia de cierta evidencia sobre que los costos relacionados con la muerte, más que el envejecimiento en sí mismo, son el principal factor detrás de estos cambios en el gasto ${ }^{8}$. Otro es que las mejoras en los niveles de bienestar que derivan en mejoras en las ingestas nutricionales, conductas preventivas y el acceso a mejores tratamientos, permitirían presuponer que frente al aumento en la esperanza de vida ${ }^{9}$ las tendencias crecientes en el gasto por este motivo deberían reducirse en el futuro ${ }^{10}$. Nótese que este último efecto se podría ver estimulado con las mejoras de salud provocadas por las políticas públicas que afectan los precios relativos de ciertos bienes no saludables, como tabaco, alcohol o azúcar, a través de alícuotas impositivas cada vez mayores.

Los cambios en el envejecimiento e ingreso afectan las condiciones de demanda, pero desde la oferta también hay una presión al aumento en los costos, originada en dos efectos: por un lado, la renovación tecnológica que lleva a un aumento tendencial del gasto ${ }^{11}$, el cual en su interior puede adoptar un signo

\footnotetext{
${ }^{1}$ La Meta 3.8 de los Objetivos de Desarrollo del Milenio (ONU (2015)) cristaliza la preocupación de las Naciones Unidas, pero también de otros organismos multilaterales (World Health Organization y World Bank (2014)).

${ }^{2} \mathrm{Xu}$ et al (2018).

${ }^{3}$ Newhouse J. P. (1977).

${ }^{4}$ Esta discusión es relevante con el objeto de saber si frente a una recesión el gasto en salud se reduce o se mantiene, con los consiguientes impactos sobre la cobertura poblacional. Baltagi y Moscone, (2010) y Baltagi et al (2017) discuten especialmente este punto.

${ }^{5}$ Fogel (2009); Chernew y Newhouse (2012); Meijer et al (2013).

${ }^{6}$ De Meijer et al. (2013).

${ }^{7}$ Deaton (2004).

${ }^{8}$ Zweifel et al. (1999).

${ }^{9}$ Deaton (2004).

${ }^{10}$ Fogel (2009).

${ }^{11}$ Di Matteo (2005)
} 
difuso dependiendo del ítem de gasto, la efectividad y costos de las incorporaciones ${ }^{12}$. Por el otro, este efecto se puede reforzar por la "Enfermedad de costos" de Baumol por la cual las diferencias de productividad de este sector respecto del promedio de la economía llevan a aumentos permanentes de los $\operatorname{costos}^{13}$.

En este contexto, surge la pregunta de por qué resultaría relevante realizar una investigación que pretenda dilucidar si la idea de que "The health sector has become one of the main sectors of the global economy, linked to economic growth, demographic change and technological change" (Xu et al (2018), pág. 3) es robusta para cualquier región socioeconómica. Esta tensión entre demografía, ingresos y tecnología con relación al gasto en salud se estudia arduamente en la literatura con diversos conjuntos de datos, países y metodologías ${ }^{14}$ en la búsqueda de conocer con precisión los valores de los coeficientes del modelo de manera de poder realizar proyecciones que permitan sustentar y evaluar políticas públicas ${ }^{15}$. Esta heterogeneidad motiva a buscar un marco unificado para disponer de resultados consistentes y comparables.

Desde lo metodológico, en este trabajo se sigue la estrategia de Di Matteo (2005) para identificar los efectos de los cambios tecnológicos, incluyendo a su vez controles que buscan tener presentes los problemas de heterogeneidad espacial y temporal como en Baltagi et al (2017). En cuanto a los datos, se utilizan datos abiertos publicados por el Banco Mundial, mientras que para la Argentina se utilizan datos del Ministerio de Economía de la Nación para el gasto, y de la base SEDLAC para ingresos, envejecimiento y presión de costos—ambas disponibles de manera pública-

Es por ello que, en el contexto de una estrategia de modelización típica macro ${ }^{16}$, se adopta un enfoque novedoso que trata de considerar esencialmente las heterogeneidades que surgen por las dimensiones no observadas. Así, primero se realiza un estudio para todos los países del mundo y se trata de establecer si este modelo es diferente por regiones. A partir de allí, se trabaja con esos mismos datos, pero limitados a América Latina, tratando de buscar una mayor homogeneidad de no observables, como cultura, religión etc. Finalmente, se da un paso adicional y se trata de evaluar qué sucede al interior de un mismo sistema: se aplica el modelo al gasto público provincial de la Argentina, el cual refleja un comportamiento similar tanto en el acceso y uso por los pacientes como en la forma en que se remunera a los insumos ${ }^{17}$.

El resto del documento se organiza de la siguiente manera. En la sección 2 se realiza una revisión de los estudios previos con relación al vínculo entre los determinantes y el gasto. La estrategia empírica empleada para realizar la evaluación de estos últimos es por medio de técnicas de datos en panel, como se detalla en la sección 3. En la sección 4 se aplica dicha metodología a datos por países provenientes del Banco Mundial para el período 2000-2015. Con ellos, se analizan las disparidades regionales a nivel mundial en los niveles de gasto y se determina la peculiaridad de América Latina frente al resto de las regiones. También se muestra que, dentro de dicha región, los países no se comportan de la misma manera, situación que no puede ser evaluada debido a la falta de información por país. Es por ello que a continuación se estudia el caso de la República Argentina, lo que permite adentrarse en un sistema único y por lo tanto de similares condiciones de demanda y de oferta, pero que puede adolecer de un proceso más lento de adopción de tecnología. Si esto fuese cierto, dados los restantes determinantes, los aumentos de gasto se explicarían sólo por problemas de productividad o de costo. Las principales conclusiones de este estudio, se sintetizan en la sección 5 . El documento culmina con las usuales secciones de referencias (6) y el anexo de tablas y figuras (7).

\footnotetext{
${ }^{12}$ Chandra y Skinner (2012)

${ }^{13}$ Baumol et al (2012)

${ }^{14}$ Ver entre otros Gerdtham y Jönsson (2000); Chernew y Newhouse (2012).

${ }^{15}$ Todos los organismos multilaterales relacionados con el sector tienen un modelo de proyecciones. Ver Marino et al (2017) para una revisión de ellos a la par de una excelente evaluación de los antecedentes y valores cuantitativos de los parámetros.

${ }^{16}$ Esta denominación sigue la utilizada en Astolfi et al (2012).

${ }^{17}$ En especial con relación a las contrataciones de recursos humanos las cuales están en el centro de la función de producción del sector (Baumol et al (2012)).
} 


\section{ANTECEDENTES}

Una idea comúnmente aceptada en los estudios sobre determinantes del gasto en salud es que su evolución depende esencialmente de tres factores: el cambio demográfico hacia poblaciones de mayor edad, la mejora en las condiciones de vida que se refleja en mayores niveles de ingreso y el aumento de costos producto de la innovación en los diagnósticos y/o tratamientos ${ }^{18}$. En esta sección se revisan estas ideas con el objeto de poder comprender las virtudes y limitaciones de las estimaciones econométricas resultantes.

Antes de realizar este análisis es importante considerar que los aspectos institucionales pueden ser un elemento fundamental de las tendencias en el gasto. De hecho, a partir del reconocimiento de su tendencia positiva, es que suelen aplicarse políticas de contención, como la forma de pago a los proveedores o la introducción de medicamentos genéricos, que no siempre son iguales. Pero convertir esta dimensión en algo cuantificable es bastante difícil: de la Maisonneuve et al (2016) asocian el efecto de las influencias estructurales con dimensiones como la regulación de la oferta de profesionales médicos, del precio de medicamentos, del médico de cabecera/familia, la definición del paquete prestacional o los objetivos de la política sanitaria, entre otras; y encuentran que ellas explican el 23\% de las variaciones en el gasto público real por persona entre países de la OECD para 2000-2011. Contar con esta discriminación de información para un conjunto más amplio de países es una tarea muy compleja. Es por esto que se trabaja con grandes paneles de países: la estrategia de datos en panel permite controlar por este efecto, pero de una manera genérica. Obviamente si la estrategia empírica se apoya en un único sistema de salud estos aspectos tienden a desvanecerse ${ }^{19}$.

Antes de continuar, debe destacarse un factor común a todos estos hechos, que es la política regulatoria en salud. La misma tiene como objetivo central establecer procedimientos con certeza sobre sus efectos sobre la salud (enfermedad), como la aprobación de protocolos de tratamientos, nuevas drogas y/o medicamentos, etc.; pero también se encarga de establecer cierta racionalidad en el conjunto de los servicios prestados a partir de las relaciones de costo beneficio ${ }^{20}$. Es por esto que resulta relevante saber cuál es el espacio posible de acción para este tipo de medidas con el objeto de contener las presiones fiscales del sector salud.

\section{II.1. ENVEJECIMIENTO Y GASTO EN SALUD}

Una idea usual en la literatura es que el gasto agregado en salud aumenta a medida que la población envejece, dado que se demandan servicios adicionales originados en la mayor esperanza de vida ${ }^{21}$ y/o por el cambio en los perfiles epidemiológicos en los que aumenta la presencia de enfermedades crónicas que, acompañados por los cambios tecnológicos, derivan en mayores usos para la misma edad y sexo ${ }^{22}$. Esta idea se apoya en resultados como los que muestra la Figura 1 que presenta Fogel (2009), en la cual se puede ver que los costos de atención médica aumentan de manera creciente a medida que aumenta la edad, al punto que se multiplica por 6 veces entre 50-54 y +85 años. Es por resultados de este tipo que un conjunto importante de estudios se ha concentrado en evaluar los cambios en la participación por tramos de edades, típicamente mayores de 65 años. Además, este tipo de indicadores resultan de sencilla incorporación a las estimaciones econométricas porque, esencialmente, su cálculo también lo es y por lo tanto suele estar disponible prácticamente de manera universal.

\footnotetext{
${ }^{18}$ Newhouse (1992).

${ }^{19}$ Estos dos representan a las situaciones que se consideran en las estimaciones econométricas: estudios globales (188 países) o regionales (América Latina) versus la relativa homogeneidad de un país (Argentina).

${ }^{20}$ Culyer (2016) discute en detalle cómo implementar una política de incorporación de tratamientos a ser cubiertos por los fondos de aseguramiento en salud a partir de la razón costos-efectividad.

${ }^{21}$ Este cambio no implica un aumento del gasto por persona individual pero sí en el agregado, ya que con todas las variables constantes hay mayor cantidad de potenciales usuarios.

${ }^{22}$ En este caso hay un aumento en el gasto por persona en proporción al aumento de la prevalencia de las enfermedades crónicas, el cual se multiplica con el aumento en la esperanza de vida.
} 
Figura 1. Distribución de la atención médica por tramo de edad en USA

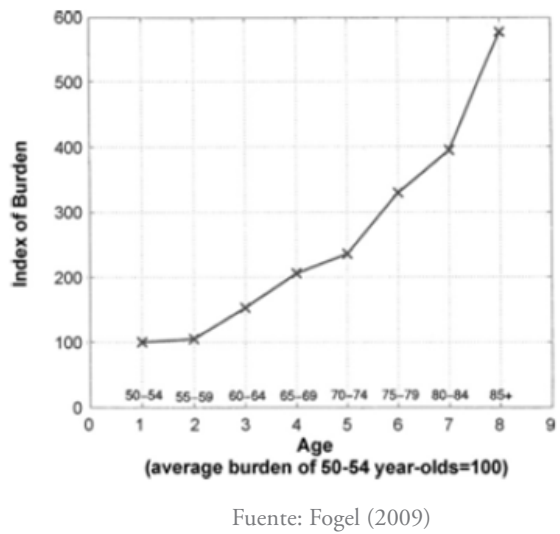

No obstante, no sería correcto aproximar el vínculo entre envejecimiento y gasto por medio de esa variable ya que la evidencia reciente indica que los individuos también llegan a mayores edades con mejor estado de salud por lo que a la misma edad hay un menor uso de prestaciones ${ }^{23}$ y por lo tanto, el efecto dominante se relaciona con el período previo al fallecimiento. Al respecto, Zweifel et al. (1999) ${ }^{24}$ muestran que el gasto aumenta significativamente en los dos años previos a la muerte y muy significativamente en los últimos 6 meses. Fogel (2009) muestra que, entre beneficiarios de Medicare, los gastos en los últimos dos años de vida representan el $40 \%$ del total del gasto del programa debido a que el costo anual es el mismo 5 años antes de la muerte a todos los costos anuales por persona, pero en el segundo año aumenta un $60 \%$ y en el ańo de la muerte crecen en promedio unas 4 veces. En el mismo sentido, Lis et al (2016) encuentran que los gastos aumentan entre 2 y 3 veces más rápido en los tres ańos previos a la muerte.

Debe observarse que estos hechos son consistentes también con la hipótesis de envejecimiento: como las personas se vuelven ancianas es más probable que mueran y, por lo tanto, el envejecimiento lleva a aumentos en el gasto simplemente en el proceso de ajuste hacia un nuevo equilibrio de edades. En los hechos estos aumentos en los gastos se deben a que, en general, el momento de la muerte se encuentra altamente asociado con la probabilidad de estar hospitalizado. Por ejemplo, Seshamani y Gray (2004) muestran que los costos aumentan linealmente a partir de los 16 ańos antes de morir, pero la probabilidad de ser hospitalizado tiene un crecimiento exponencial durante ese período con el efecto de cuadriplicar al gasto desde el penúltimo al último año de vida.

Entonces, el uso de la variable de proporción de población con más de 65 años como indicador de esta relación puede ser útil, pero lo más probable es que su significatividad converja a cero ${ }^{25}$. Esto se debe a que la variación temporal de la misma es muy pequeña a la par que el efecto está incorrectamente medido, salvo que la tecnología esté comprimiendo a la población en una única edad de muerte ${ }^{26}$. Debe notarse que en la transición hacia un nuevo equilibrio demográfico es muy probable que, en la medida en que la masa de frecuencia se concentre en torno al valor de esperanza de vida, el gasto tienda a aumentar, pero debido a que aumenta la frecuencia de episodios cercanos a la muerte en este grupo poblacional, no a que envejecen (lo cual también sucede). Estos resultados motivan pensar si es correcto asociar al envejecimiento con el gasto total en salud (público y/o privado) o si en realidad debería vincularse con otras clasificaciones como el gasto en cuidados de largo plazo.

\footnotetext{
${ }^{23}$ Fogel (2009) considera que esto se debe a las mejoras en las conductas nutricionales y los mejores tratamientos médicos.

${ }^{24}$ Esta idea se ha desarrollado en varios estudios como Aprile (2007), Breyer y Felder (2006) y Yang et al (2003).

${ }^{25}$ Lis et al. (2016).

${ }^{26}$ Breyer y Felders (2006)
} 


\section{II.2. INGRESOS Y GASTO EN SALUD}

Los ingresos afectan los gastos en salud, como en la mayoría de los bienes, al afectar su demanda tanto en las mayores cantidades como también por el aumento en las preferencias por la calidad y tipo de bienes. En términos agregados, este cambio en las preferencias presiona sobre los fondos financiadores de servicios de atención médica, los cuales expanden las prestaciones que son financiadas con los ingresos adicionales de dichos fondos por el mayor nivel de ingreso ${ }^{27}$. En general, las estimaciones econométricas tratan de establecer el valor de la elasticidad ingreso del gasto, la cual se espera se ubique por debajo de 1, indicando que se trata de un bien necesario ${ }^{28}$. Esto implica que también va a moverse en sentido directo con el nivel de desarrollo: un mayor desarrollo implicaría una mayor elasticidad ${ }^{29}$. Pese a la extensa y variada literatura, como lo demuestra la revisión de Baltagi et al (2017), persiste la discusión sobre el valor exacto de este parámetro.

Obviamente no se trata de un tema nuevo, siendo sólo algunos antecedentes históricos el trabajo de Kleiman (1974) o el de Getzen (1992) en el contexto de la discusión sobre envejecimiento. Los primeros estudios con datos cross-section para países de la OECD estiman una elasticidad ingreso superior a 1. Las estimaciones eran de $(1,2 ; 1,5)$ con la excepción de algunos como Parkin et al. (1987) que obtiene un valor de $0,9^{30}$. Estos estudios tienen varios sesgos al omitir las características idiosincráticas de cada país y momento. El inicio de los 2000 marcó la transición hacia los estudios de paneles que en algunos casos incluyen test de raíz unitaria, co-integración y estacionalidad, e incluso algunos realizan paneles dinámicos, como en Holly et al. (2011).

Aunque la evidencia reciente sugiere que la atención médica puede ser una necesidad ${ }^{31}$, el patrón de mayores elasticidades a mayores niveles de ingreso se observa cuando el enfoque se desplaza de paneles nacionales a internacionales y cuando se comparan los paneles internacionales de unos pocos países con los únicos dos estudios de más de 130 países (Holly et al. (2011) y Farag et al. (2012)). Pero la relación entre ambos no es lineal, ya que de esos estudios surge que la distribución de la elasticidad ingreso no es lineal entre países, sino que sigue una forma general por la cual los países de ingresos bajos y altos tienen una elasticidad mayor que los de ingresos medios.

Todos estos antecedentes se basan en obtener estimaciones a partir de un panel de países. Sin embargo, algunos de estos estudios se limitan el análisis a un único país, por distintos motivos. Por un lado, Getzen (2000) argumenta que hay una tendencia a que el ingreso se vuelva un bien de lujo a elevados niveles de agregación. Por el otro, en muchos países desarrollados los individuos pueden tener una elasticidad ingreso igual a cero debido a los esquemas de aseguramiento que diluyen o remueven los gastos de bolsillo ${ }^{32}$ y así, el gasto agregado no sigue necesariamente el mismo patrón de comportamiento. Es por esto que estudiar los resultados al interior de un país podría generar intuiciones interesantes, pero dada la cantidad de observaciones requeridas en la estimación, resulta de difícil logro. Por lo tanto, como se hace en este trabajo para el caso de Argentina, poder avanzar sobre esta situación sería un aporte interesante a la literatura.

\footnotetext{
${ }^{27}$ En los hechos, estos aumentos son por mayor salario que deriva en mayores impuestos y/o contribuciones a la seguridad social.

${ }^{28} \mathrm{La}$ idea de bien necesario resulta clara cuando se piensa en que una reducción del ingreso, si el bien es necesario, implicaría que la demanda del mismo se reduciría menos que proporcionalmente. Como resultado, es un gasto cuya evolución tiende a ser más bien de rígida.

${ }^{29}$ Esto implica que las preferencias agregadas también están cambiando a medida que el ingreso aumenta.

${ }^{30} \mathrm{La}$ única diferencia de este estudio con los restantes es que utiliza datos deflactados por paridad de del poder adquisitivo.

${ }^{31}$ Tanto es así que, en términos generales, los organismos multilaterales utilizan diversos valores para sus proyecciones: la OECD utiliza un promedio de 0,8 con un intervalo de entre 0,6 y 1 para los estudios de sensibilidad; el IMF utiliza 0,3 y el CBO (USA) utiliza 1 (Marino (2017))

${ }^{32} \mathrm{Tal}$ es el caso, sólo a modo de ejemplo, de UK o Francia.
} 


\section{II.3. TECNOLOGÍA Y GASTO EN SALUD}

El cambio tecnológico ha sido identificado como aquella variable que motiva de forma continua y más rápidamente los movimientos del gasto en salud ${ }^{33}$. El avance tecnológico se canaliza de varias formas: como productos, conocimiento o procesos; por lo que, las nuevas tecnologías pueden extender el grado, el rango y la calidad de los servicios de salud y así, su efecto sobre el gasto no es claro ni directo ${ }^{34}$. Mientras que algunas pueden ahorrar recursos, otras pueden aumentar los costos al ofrecer mejores (pero más complejos) servicios para enfermedades complejas e incluso resolver algunas que previamente eran intratables. En este sentido, es importante no montar una contienda en contra de las nuevas tecnologías, ya que podría suceder que sean altamente efectivas con poca posibilidad de sobreuso como una terapia retroviral de HIV 35 . Algunas tecnologías reducen el tiempo de espera en la resolución porque permiten aumentar el volumen de prestaciones (nuevos test que diagnostican mejor y más rápido). Otras, en cambio, pueden alargar la espera (son más precisos, pero más lentos o más caros). Las incorporaciones especialmente preocupantes en términos de la expansión del gasto son aquellas cuyos beneficios son pequeños o con poca evidencia ${ }^{36}$ ya que a la problemática fiscal agregan el desacople con los resultados en salud.

Una cuestión no menor es que la tecnología y el envejecimiento suelen modelarse de manera separada, cuando es posible que los cambios en la esperanza de vida sean resultado de las mejoras en los tratamientos médicos (especialmente de aquellos asociados a los cuidados de largo plazo), que junto con otros factores como el cambio en la alimentación o el abandono del tabaco, llevan a que las personas puedan envejecer en mejores condiciones de salud y vivir más tiempo ${ }^{37}$. Este aumento en la cantidad y calidad de los servicios de atención médica no sólo modifica los resultados en salud, sino que también podría afectar los niveles de gasto. Esta pequeña discusión sobre el vínculo entre tecnología y gasto muestra que esta dimensión es muy compleja como para poder definir en términos empíricos una simple variable que caracterice la relación entre estas variables. La evidencia sugiere que, en promedio, la tecnología tiene un efecto positivo sobre el gasto, aunque hay que tener presente que se trata de una suma de valores positivos y negativos según la definición de tecnología que se adopte. Además, dependiendo del método utilizado para medir el cambio, la estimación puede incluir a las políticas y otras variables que confunden el efecto.

En la literatura se han utilizado dos enfoques para medir el cambio tecnológico ${ }^{38}$ : uno, basado en lo difuso de la relación entre las variables, es asumir que todo aquello que no es explicado por el ingreso y/o envejecimiento se debe a la tecnología ${ }^{39}$. Este método es relativamente sencillo y es usual cuando no se disponen estimaciones de tecnología o son muy endógenas como para utilizarlas ${ }^{40}$. Pero, si en el modelo explicativo de envejecimiento e ingresos se incluye una (o un conjunto de) variable(s) para la tecnología y con una buena capacidad explicativa, ambos métodos convergen a devolver resultados similares, con la ventaja de que este último permite disponer de un coeficiente definido para la tecnología.

El otro enfoque para incluir la tecnología es utilizar una dimensión/variable específica como tasas de mortalidad, esperanza de vida, gastos en R\&D, patentes o similares. Obviamente, estas dimensiones no capturan completamente el fenómeno, ya que el problema de la tecnología es que no puede ser apropiada completamente por aquel que la generó, entonces un índice adecuado sería aquel que permita reflejar la

\footnotetext{
${ }^{33}$ Chernew y Newhouse (2012).

${ }^{34}$ Chernew y Newhouse (2012).

${ }^{35}$ Es tecnología Tipo I en la clasificación de Chandra y Skinner (2012).

${ }^{36}$ Tipo III según Chandra y Skinner op. cit.

${ }^{37}$ Fogel (2009).

${ }^{38}$ Ver en análisis más detallado en Chernew y Newhouse (2012)

${ }^{39}$ Este enfoque se deriva de una reflexión análoga sobre la idea del residuo de Solow (1957) con relación a la explicación de los procesos de crecimiento económico.

${ }^{40}$ Holly et al. (2011); de la Maisonneuve y Oliveira Martins (2013)
} 
adopción de nuevas tecnologías sanitarias. De todas maneras, adoptar los resultados sanitarios como indicador de su aplicación tampoco es correcto, ya que muchos de los cambios en ellos se deben a otras cuestiones, en especial en países en desarrollo y/o pobres, como la disponibilidad de agua potable o vivienda adecuada. Es por esto que algunos autores ${ }^{41}$ proponen analizar caso por caso su efecto sobre el gasto, estrategia que no permite realizar una evaluación de la pregunta macro sobre el vínculo entre gasto y tecnología. Al igual que en el caso del ingreso, una variación de esta estrategia podría ser realizar estudios a nivel de país. Por ejemplo, You y Okunade (2017) encuentran, para Australia, que varias de estas variables son significativas; y que un indicador del instrumental médico tiene signo mayor que el gasto en $\mathrm{R} \& \mathrm{D}$, lo cual probablemente se deba al desacople temporal de esta última variable.

Una alternativa es considerar un enfoque intermedio entre los mencionados anteriormente. Esto es, asumir que el efecto de la tecnología es aumentar el gasto y, por lo tanto, buscar una variable exógena que tenga tendencia positiva. Claramente, si se pudiese encontrar tal variable la misma adolecería de que todos los efectos de variables no observadas con signo positivo serían subsumidos en su coeficiente. Di Matteo (2005) utiliza el tiempo como indicador de tecnología, el cual además de la deficiencia comentada también puede ser criticada por la contemporaneidad de los efectos: posiblemente la introducción de una nueva droga tenga un efecto temporal sobre el gasto que es inmediato mientras que un nuevo equipo es posible que su efecto se vaya difundiendo en el tiempo.

No obstante, en las estimaciones econométricas de este trabajo se utilizará ese criterio bajo dos ideas. La primera, es la ausencia de medidas alternativas a una estrategia utilizada en la literatura ${ }^{42}$, por lo que omitirla sesgaría los resultados y les quitaría intuición. Esta premisa se basa en la segunda idea de que resulta difícil encontrar un proceso que sistemáticamente afecte a todos los países a lo largo del tiempo en el mismo sentido. Así, el efecto "tecnología” que captura estas estimaciones es aquel proceso sistemático y similar al conjunto de países o regiones que empuja hacia arriba el gasto más allá de los efectos que tienen las otras variables.

Otro problema relacionado con la tecnología es el aumento de costos originado en las características de este sector ${ }^{43}$ por las diferencias en la evolución de la productividad sectorial respecto del promedio de la economía (Baumol, 1967). Esta idea, conocida como "Enfermedad de Costos", cuya aplicación suele ser sobre el conjunto sectorial y no sólo sobre el sector público ${ }^{44}$, implica que para retener a los trabajadores en el sector se requieren aumentos salariales por encima del promedio de la economía ${ }^{45}$. Este hecho se debe a la función de producción y su relación con la tecnología, que impiden realizar grandes ganancias de productividad: es muy difícil sustituir a los profesionales que diagnostican. Además, el efecto se ve reforzado por las nuevas tecnologías, cuya aplicación requiere de profesionales más capacitados y por lo tanto mayores salarios para compensar los ańos invertidos en estudios.

Los precios relativos (más bien su evolución) también podrían ser candidatos para reflejar este efecto, pero debido a que no suelen reflejar bien la calidad, no son utilizados. Aun cuando se utilice el deflactor implícito del PBI, las diferencias metodológicas y de composición hacen difícil de utilizar estas variables en un estudio que compare países. Además, como argumenta Hartwig (2008), los gobiernos tienen la posibilidad de influenciar el gasto en salud en formas diversas, y en un ambiente de precios muy regulados hay formas de desplazar los recursos hacia este sector sólo aumentando los precios ${ }^{46}$. En este marco, Hartwig (2011) analiza esta hipótesis con un deflactor de precios médicos, el cual tiene un coeficiente positivo en todas las especificaciones, con un rango de 0,38 a 0,6 y controlando por ingresos.

\footnotetext{
${ }^{41}$ Como por ejemplo en Chernew y Newhouse (2012)

${ }^{42}$ Di Matteo (2005) o Baltagi y otros (2017).

${ }^{43} \mathrm{Y}$ también de otros con funciones de producción similares, como en educación o cultura. Al respecto, véase Baumol (2012)

${ }^{44}$ Hartwig (2008); Colombier (2012).

${ }^{45}$ Esta idea se propone en Baumol (1967) y es analizada empíricamente en Hartwig (2008, 2011) o Colombier (2012).

${ }^{46}$ Hartwig (2008).
} 
Es por esto que, a partir de Hartwig (2008), se utilizan los salarios relativos del sector respecto del conjunto de la economía ${ }^{47}$. Este coeficiente tiene la ventaja de ser sencillo y homogéneo en su cálculo, aunque se suele argumentar que los problemas de calidad no se ven reflejados, por lo que habría que ver qué sucede con las diferencias de capital humano. Este parámetro fluctúa, en los valores promedios, entre 0,19 (Colombier, 2012) y 0,96 en Medeiros y Schwierz (2013) o 0,85 en Hartwig (2008). Desafortunadamente, no se dispone de un indicador de este tipo para todos los países en el tiempo, pero sí para el caso de las provincias de la Argentina, por lo que las estimaciones para ellas incluirán el efecto de empuje de costos de Baumol.

\section{METODOLOGÍA Y DATOS}

Para tratar de entender cómo se relacionan las variables consideradas en la sección anterior, se construyen dos paneles de datos. El primero, internacional, surge de datos provenientes del Banco Mundial ${ }^{48}$ para el período 2000-2015, para el cual hay datos disponibles de gasto público en salud a paridad de poder de compra para 186 países $^{49}$. El análisis con este conjunto de datos tiene dos virtudes respecto de los estudios previos: por un lado, se extiende la cobertura temporal respecto de Baltagi et al (2017), quedando los eventos de la crisis internacional de 2008 incluidos en el período de estudio. Por el otro, se trata de una revisión que realizó la OMS sobre una nueva edición del sistema de cuentas nacionales en salud, la cual tiene mayor precisión y se encuentra enfocada en el logro de la cobertura universal en salud ${ }^{50}$.

En la Tabla 1, que presenta y caracteriza las variables que conforman el panel internacional, puede verse que el gasto público por persona mundial a paridad del poder adquisitivo (PPP, por sus siglas en inglés) más que se duplicó entre 2000 y 2015. Los datos sugieren que no es solo la población mayor de 65 años la que "empujaría" los costos de los sistemas de salud, sino también la población en edad activa. No obstante, como se ha considerado previamente, no sería de esperar un coeficiente muy elevado ya que, en estos 15 años, en promedio, la proporción de población mayor de 65 años aumentó 1,5 puntos, es decir, un 22\%. Por otro lado, se observa que el producto por persona evoluciona a una tasa menor que el gasto, pero mayor que el envejecimiento: una estimación grosera de dicha elasticidad diría que cuando el producto aumenta el gasto lo hace más que proporcionalmente, o sea, una elasticidad ingreso mayor que uno. Claramente, las tasas de crecimiento de estas dos variables sugieren que hay otro factor explicando el gran aumento del gasto.

Tabla 1. Definición de variables y estadísticas descriptivas, 2000-2018

\begin{tabular}{lcccc} 
& 2000 & 2005 & 2010 & 2015 \\
\hline Gasto per capita PPP & 645,9 & 853,9 & 1103,3 & 1366,3 \\
Prop. 0-14 años & 32,6 & 30,5 & 28,9 & 27,9 \\
Prop. 15-64 años & 60,6 & 62,2 & 63,4 & 63,7 \\
Prop. +65 años & 6,9 & 7,3 & 7,7 & 8,4 \\
GDP per capita PPP & 11831,4 & 14672,6 & 17609,5 & 20086,4 \\
América Latina y Caribe & 0,2 & 0,2 & 0,2 & 0,2 \\
Asia E. & 0,2 & 0,2 & 0,2 & 0,2 \\
N. Africa & 0,1 & 0,1 & 0,1 & 0,1 \\
América del Norte & 0,0 & 0,0 & 0,0 & 0,0 \\
Europa y Asia Central & 0,3 & 0,3 & 0,3 & 0,3 \\
Asia S. & 0,0 & 0,0 & 0,0 & 0,0 \\
Africa SS. & 0,2 & 0,2 & 0,2 & 0,2 \\
\hline Fuente: Elaboción
\end{tabular}

Fuente: Flaboración propia sobre la base de Banco Mundial (https://datos.bancomundial.org/)

Nota: Las estimaciones de población son realizadas por personal del Banco Mundial sobre la base de la distribución por edades/sexo de las Previsiones Demográficas Mundiales de la División de Población de las Naciones Unidas.

\footnotetext{
${ }^{47}$ Con algunas variaciones, se aplica en Colombier (2012), Medeiros y Schwierz (2013); Ho y Zhou (2014); Bates y Santerre (2013).

${ }^{48}$ Los cuales se descargan utilizando el comando de Stata wbopendata. Detalles adicionales sobre este comando se pueden ver en https://datahelpdesk. worldbank.org/knowledgebase/articles/889464-wbopendata-stata-module-to-access-world-bank-data En el caso de este trabajo, el comando utilizado es: wbopen-data, indicator(ny.gdp.pcap.kd.zg; ny.gdp.pcap.pp.cd; sp.pop.65up.to.zs; sp.pop.1564.to.zs; sp.pop.0014.to.zs; sh.xpd.chex.pp.cd; sp.dyn.le00.in) long language(es) clear

${ }^{49} \mathrm{El}$ gasto privado en salud no se ha incorporado al estudio por falta de disponibilidad de datos para un panel de una magnitud tal como el incorporado en este trabajo.

${ }^{50}$ Sobre el sistema de cuentas nacionales se puede consultar: OECD/Eurostat/WHO (2017) o https://www.who. int/health-accounts/methodology/en/
} 
Detrás de este promedio mundial hay diferentes comportamientos regionales. En la Figura 2 se presenta la evolución del gasto en salud por persona (PPP) por región. Allí puede verse que el nivel de gasto por persona aumenta con el nivel de desarrollo. A los objetivos del problema, para América Latina se puede ver que la región se ubica entre los menores niveles de ingreso con una tendencia relativamente estable. Si a este hecho se le incorpora la necesidad de mejores logros sanitarios por mejoras en la efectividad y en el acceso, es posible comprender por qué los responsables regionales de las políticas sectoriales se encuentran preocupados por la posibilidad de lograr los recursos fiscales necesarios ${ }^{51}$. En este sentido, es interesante notar que el problema de aumento del gasto pareciera ser más un hecho de los países desarrollados que de aquellos de ingresos medios o bajos, en un contexto en el cual todas las regiones muestran una tendencia, aunque sea muy leve, que es creciente. Este hecho también sugiere que posiblemente el modelo explicativo base, en el cual la presión de costos es un factor clave, sea una buena explicación subyacente al hecho de que el gasto por persona aumenta más que el ingreso y el porcentaje de población de mayor edad.

Figura 2. Gasto Público y sus determinantes: Evolución por regiones. 2000-2015

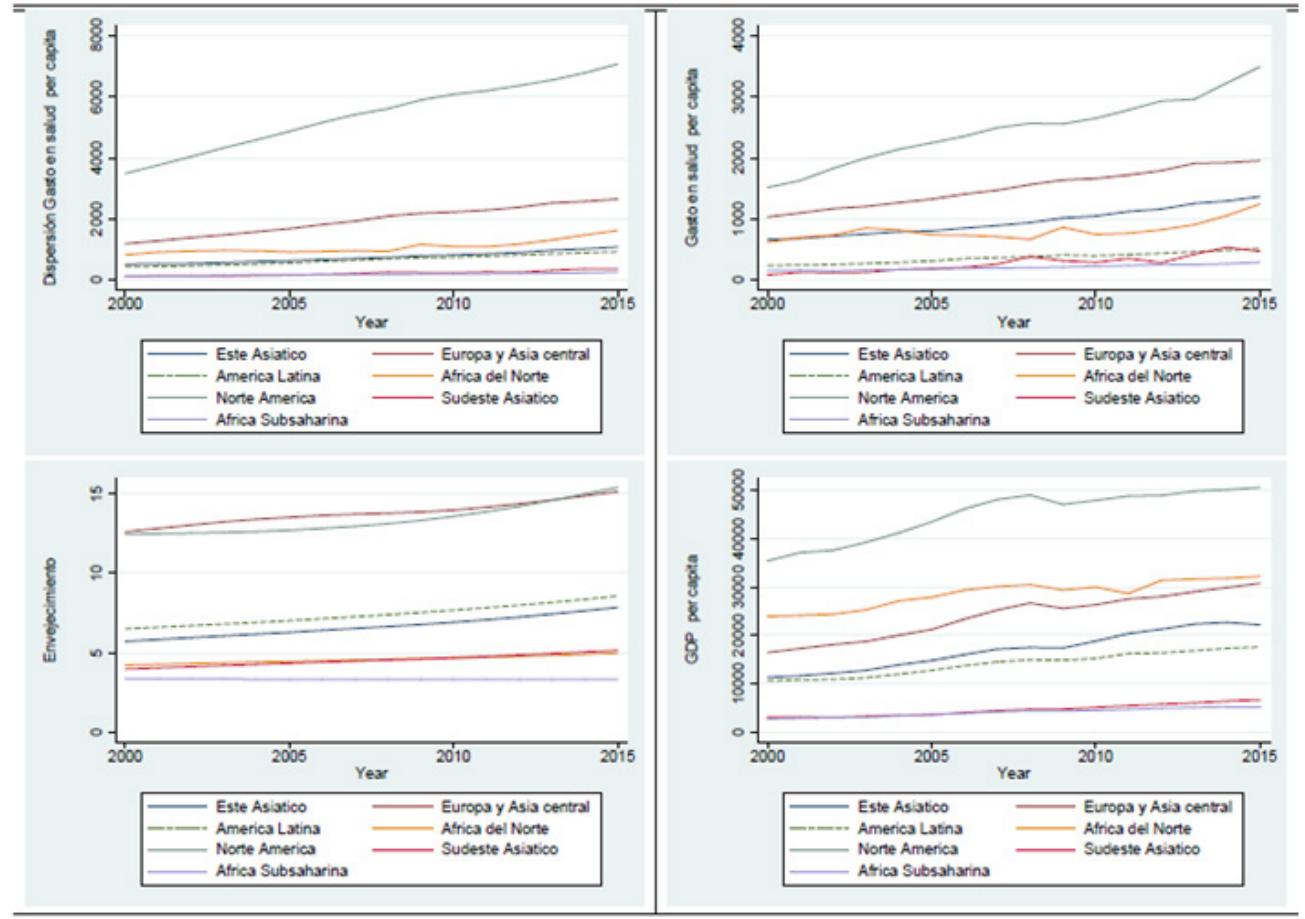

Fuente: Elaboración propia sobre la base de Banco Mundial (https://datos.bancomundial.org/)

En este contexto, la pregunta entonces es: ¿̨cuáles son los determinantes del gasto en salud por persona? en especial si las cuestiones relacionadas con envejecimiento e ingreso son diferenciales por regiones. Para ello, se estiman con la técnica de paneles con efectos fijos diversas especificaciones de este modelo general por el cual, para cada país $i$ en el momento $t$, el logaritmo del gasto por persona $\left(y_{\mathrm{i}}\right)$ depende del logaritmo del envejecimiento (logaritmo del \% de población mayor de 65 años), del logaritmo del nivel de ingreso (logaritmo del producto por persona en PPP) y de la tecnología (capturada por el paso del tiempo). La ecuación a continuación formaliza el modelo:

$$
y_{\text {it }}=\alpha+\beta * \text { envejecimiento }_{\text {it }}+\eta * \text { ingresos }_{\text {it }}+\gamma * \text { tecnologia }_{t}+\varepsilon_{\text {it }}
$$


Las variables envejecimiento y tecnología se aplican con las siguientes aclaraciones. En cuanto a la demografía, los modelos básicos también se estiman incluyendo como control a la población en edad activa (\% de población entre 14 y 64 años) por lo que en este caso los coeficientes deben leerse con referencia al grupo base de los menores de 14 años. La tecnología se implementa de la siguiente manera:

¿Por qué se adopta esta modelización? El principal problema en la aplicación empírica es que todas las variables asociadas a la tecnología son difíciles de obtener para paneles con el tamaño aquí considerado. Este hecho concreto, sumado a las dificultades para entender los canales por los que se propagan los efectos, sugieren seguir la estrategia de Di Matteo (2005) con una tendencia creciente ${ }^{52}$. Aquí la idea es que existe algún proceso exógeno, no relacionado con el ingreso ni con la demografía, que empuja de manera permanente y sistemática al gasto. A este proceso se lo denomina tecnología.

La ausencia de variables que capturen las diferencias en los sistemas de salud y el nivel de ejecución del gasto público entre países y en el tiempo constituye una limitación al análisis realizado.

El segundo panel surge de datos exclusivos de la República Argentina para el período 2003-2015 de la ejecución de gasto público por parte de los gobiernos subnacionales ${ }^{53}$. Antes de proseguir con su descripción, es importante notar que estos gobiernos provinciales son quienes ejecutan gran parte del gasto público en atención médica del país ${ }^{54}$. Este panel permite mejorar la precisión de la información sobre gasto público con respecto al panel de países. Del lado de la variable independiente, los datos de gasto público surgen de los procesos contables del Ministerio de Economía de la Nación, por lo cual se suponen relativamente homogéneos. Adicionalmente, se reflejan actividades de sistemas de salud locales con organización relativamente similar ${ }^{55}$ por lo que, en esencia, funcionan bajo un esquema similar de incentivos para prestadores ${ }^{56} \mathrm{y}$ usuarios, quedando las peculiaridades estructurales (aquellas que no varían con el tiempo) subsumidas en el efecto fijo asociado a cada provincia. La Tabla 2 expone las estadísticas descriptivas usuales de este panel.

Tabla 2. Definición de variables y estadísticas descriptivas, regiones de Argentina 2003-2015

\begin{tabular}{lcccc} 
& $\mathbf{2 0 0 3}$ & $\mathbf{2 0 0 9}$ & $\mathbf{2 0 1 5}$ & $\mathbf{2 0 0 3 - 2 0 1 5}$ \\
\hline Gasto en salud real porc. ingreso & 1,2 & 1,4 & 1,8 & 1,5 \\
Gasto en salud real per capita & 407,0 & 1090,1 & 2573,2 & 1191,3 \\
Ing. p.c. familiar real & 324,7 & 775,6 & 1429,6 & 803,7 \\
Prop. pob. mayor de 60 años & 9,2 & 10,1 & 11,3 & 10,1 \\
Edad activa & 56,7 & 57,9 & 57,9 & 57,6 \\
Prop. pob. 61-70 años & 4,7 & 5,4 & 6,3 & 5,4 \\
Prop. pob. 71-80 años & 3,1 & 3,2 & 3,4 & 3,2 \\
Prop. pob. +80 ańos & 1,4 & 1,5 & 1,6 & 1,5 \\
Enfermedad de costos por ingresos totales & 1,4 & 1,4 & 1,3 & 1,4 \\
Enfermedad de costos por salarios horarios & 1,4 & 1,4 & 1,3 & 1,3 \\
Población sin seguro de salud & 42,9 & 33,0 & 28,6 & 34,5 \\
\hline
\end{tabular}

Fuente: Elaboración propia sobre la base de datos de la Secretaría de Hacienda de la Nación y SEDLAC (CEDLAS y The World Bank)

Una desventaja es que el gasto se encuentra expresado en valores nominales en un período de fuerte aumento del nivel precios, por lo que requiere ser ajustado a valores reales ${ }^{57}$. Esto, para el caso de Argen-

${ }^{52} \mathrm{El}$ supuesto bajo el cual se considera a la tecnología igual entre países se desprende de las mismas limitaciones que justifican la elección de la metodología citada. Incorporar una variable que permita distinguir el factor tecnológico entre países resultaría interesante para superar esta limitación.

${ }^{53}$ Estos datos se obtienen de la Direción Nacional de Asuntos Provinciales - M. Hacienda de la Nación https: //www.argentina.gob.ar/hacienda/sechacienda/ asuntosprovinciales

${ }^{54}$ En Crosta (2018) se puede ver una descripción de la evolución y estructura del gasto público en salud de la Argentina en este período. Es importante tener presente que se excluyen los llamados seguros sociales como INSSJyP o OSP. No se incorpora en el análisis el gasto en salud que el gobierno nacional realiza en las provincias, con lo cual resultaría interesante ampliar el campo de estudio para conocer sus implicancias.

${ }^{55} \mathrm{Se}$ diferencian por la presencia de gobiernos locales a nivel de pequeńas regiones o municipios. Si bien podría estimarse un modelo incluyendo una variable que refleje esta situación, lo cierto es que la mayor proporción de prestaciones de alta complejidad y de gran volumen se realizan en el nivel provincial. ${ }^{56} \mathrm{Si}$ bien es cierto que las provincias presentan heterogeneidades respecto de la forma en que contratan recursos humanos (ver Maceira et al (2017)) el mecanismo básico de contrato permanente es predominante, lo cual sugiere que se cumplen los patrones básicos de contratación de empleo público. Luego, la profesión y la organización de los sistemas locales ajustan otras formas de remuneraciones con el objetivo de cubrir cuestiones como las guardias, suplencias, etc.

${ }^{57}$ En Cetrángolo et al (2015) se puede ver una revisión de la evolución macroeconómica de la Argentina en este período. 
tina, implica una dificultad adicional ya que no se dispone de una serie de precios que refleje diferencias regionales, y de hecho en cierto período no se dispone de información alguna. Es por esto que se decide convertir los valores en unidades de la canasta básica para la medición de la pobreza ${ }^{58}$, índice que no solo está disponible para todos los años, sino que es muy utilizado en los estudios de pobreza ${ }^{59}$. Por otro lado, permite tener en cuenta las diferencias de precios relativos de las distintas regiones.

Los datos de los determinantes surgen de la base SEDLAC, la cual estima con una metodología homogénea entre años (y países) diversos indicadores demográficos, económicos y sociales, para el caso de Argentina sobre la base de microdatos correspondientes a la EPH. En este caso, los datos también se restringen al período 2003-2015 ya que, si bien la encuesta se releva desde mediados de los años 70, la composición geográfica y metodológica cambia de manera sustancial a partir de $2003^{60}$. Esta fuente de información releva la situación de los principales aglomerados urbanos del país, lo cual genera un sesgo sobre las posibles diferencias urbano-rural. No obstante, como el $95 \%$ de la población argentina vive en aglomerados urbanos, se estima que dicho sesgo es pequeño. Sobre las variables en sí, sólo resta aclarar que el ingreso resulta de la suma de los ingresos de cada uno de los hogares y no es el producto bruto geográfico, por lo que, a la par de que muestra cómo cambian las condiciones de demanda de los hogares, también pierde intensidad como indicador de la capacidad recaudatoria del gobierno, aunque la base tributaria de la Argentina son los impuestos al consumo. Obviamente, también es deflactado en la misma moneda que el gasto, o sea, unidades de líneas de pobreza.

Por otro lado, al disponer de los microdatos, es posible incorporar dos medidas para la "Enfermedad de Costos”. Siguiendo a Hartwig (2008), se computa:

$$
\mathrm{EC}_{\mathrm{it}}=\frac{w_{\mathrm{it}}^{S}}{w_{\mathrm{it}}^{E}}
$$

En donde $w^{\mathrm{S}}$ es el salario promedio en salud y $w^{\mathrm{E}}$ es el salario en el conjunto de la economía, incluyendo salud. De nuevo, dado que se disponen de los microdatos, es posible también computar dos medidas de salario: una es el salario mensual y la otra el salario horario. Si la hipótesis fuese una cuestión de productividad, sería de esperar que el salario horario tuviese una relación más intensa con los gastos. Estos indicadores se computan sobre el conjunto de los ocupados en el sector (tanto privados como públicos) con el fin de evitar posibles peculiaridades que surjan de las pujas entre los sindicatos y el gobierno, pero también por cuestiones de confiabilidad estadística ${ }^{61}$.

$$
y_{\mathrm{it}}=\alpha+\beta * \text { envejecimiento }_{\text {it }}+\eta * \text { ingresos }_{\mathrm{it}}+\gamma * \text { tecnologia }_{t}+\rho * \mathrm{EC}_{\mathrm{it}}+\varepsilon_{\mathrm{it}}
$$

Además de las aclaraciones previas, estos modelos se estiman utilizando una forma funcional más flexible de la tecnología, con la incorporación de variables dummy que reflejan cada uno de los años. De esta manera, el concepto de tecnología cambia levemente: sigue siendo un proceso sostenido de cambio, pero se le da la posibilidad de adoptar valores positivos o negativos. También es posible contar con algo más de flexibilidad en la demografía, ya que ahora se puede computar para cada provincia el ratio de grupos más pequeños de edades, y de esta manera aproximar mejor la idea de aumento de costos frente al evento de la hospitalización previa a la muerte.

${ }^{58}$ Se realizaron estudios de comparación de la evolución de estas canastas con los salarios del sector salud, con el capítulo "Salud" y el "Nivel General" del Índice de Precios al Consumidor, todos los cuales muestran una tendencia compartida y, por lo tanto, deflactar por este indicador resulta en tendencias similares. ${ }^{59} \mathrm{Al}$ punto de ser insumo de una de las principales fuentes de información sobre las condiciones socio económicas para América Latina, como la base SEDLAC http://www.cedlas.econo.unlp.edu.ar/wp/en/estadisticas/sedlac/

${ }^{60}$ Sobre las características de esta encuesta de hogares, al igual que el acceso a los microdatos, ver: https://www. indec.gob.ar/indec/web/Institucional-Indec-BasesDeDatos ${ }^{61}$ La fuente de información está diseńada para obtener datos confiables a cierto nivel de agregación. Por lo tanto, cuando la sometemos a presiones buscando porciones menores de la población, sería de esperar que aumenten los intervalos de confianza de los estimadores. 


\section{RESULTADOS}

En lo que sigue, se procede a reportar los resultados que surgen de estimar las ecuaciones (1) y (4) expresadas en términos de logaritmo de manera de poder dar cuenta de la comparación usual con la literatura en términos de elasticidades.

\section{IV.1. ANÁLISIS GLOBALES}

En esta sección se analizan los resultados de aplicar la metodología descripta anteriormente. En la Tabla 3 se presentan las estimaciones de la ecuación (1) para el conjunto de países (en las primeras dos columnas), y luego para distintas regiones (en las columnas sucesivas) ${ }^{62}$. La estimación de este tipo de modelos utilizando efectos fijos resulta una práctica habitual puesto que es una técnica que permite capturar las características idiosincráticas de cada país. No obstante, se procedió a realizar un Test de Hausman (1978) para el modelo básico, el cual arrojó una Prob $>\mathrm{chi}^{2}=0,000$ avalando la elección intuitiva de efectos fijos por sobre efectos aleatorios.

En base a la mencionada Tabla 3, entonces, podemos plantear algunos interrogantes: primero ¿cuál es el ajuste global del modelo? o ¿cuán válida en términos generales es la ecuación (1)?. Para ello, se analiza el grado de ajuste o relevancia conjunta del modelo $\left(\mathrm{R}^{2}\right)$ y el valor general de los estadísticos $\mathrm{t}$ (todas las variables resultan significativas). Otra pregunta interesante es ¿en qué medida los coeficientes obtenidos y su significancia estadística concuerdan con la literatura? Dado que ésta última es condición necesaria para la primera, se comienza por ella.

Para el conjunto de países analizados en el período 2000-2015, ¿cómo se relaciona el gasto con el envejecimiento, los ingresos y la tecnología? Las dos primeras columnas de la Tabla 3 muestran estos resultados. En ellas puede verse que las tres variables son significativas, incluso cuando se incluye a la población activa (la cual no es estadísticamente significativa). Los signos y valores de los determinantes son los esperados, en especial respecto de la elasticidad ingreso. Un comentario que merece la pena volver a realizarse es que en este modelo la comprensión de la constante es complicada, ya que todos los efectos sistemáticamente positivos se los lleva la tecnología. En cuanto a la capacidad de explicar las variaciones en términos globales, el valor del $\mathrm{R}^{2}$ es superior a 0,9 , por lo que efectivamente las decisiones respecto del gasto público en salud refieren a las tres variables aquí consideradas.

Las columnas sucesivas muestran los resultados de estimar el modelo para las distintas regiones ${ }^{63}$. Curiosamente, cuando se realiza esta desagregación surge que, de todos los efectos, el que presenta la variabilidad más notable es el envejecimiento, ya que de la revisión de la literatura sabemos que la elasticidad ingreso es variable. Respecto de la primera variable, surge que sólo es significativa en América del Norte, Asia del Este y en ambas regiones africanas, con mayor intensidad en éstas últimas. Para las restantes regiones, el coeficiente no es significativo. Este resultado es novedoso, ya que en general se suele decir que el problema del envejecimiento es de los países más desarrollados, cuando los resultados para África revelarían que no es así. No obstante, ya hemos visto que el surgimiento de coeficientes no significativos es usual en la literatura, a la par que sería de esperar (por su relativa estabilidad en el tiempo e incluso entre países) un valor bajo. Además, el vínculo no es con la estructura de edades sino con los gastos relacionados con los momentos previos a la muerte. Lo interesante de este análisis es que la fortaleza de los vínculos en esas regiones lleva a afirmar que el problema del envejecimiento es global cuando se trata de una problemática regional.

${ }^{62}$ La clasificación de regiones es la que utiliza el Banco Mundial

${ }^{63}$ Como ya se ha dicho, se sigue la clasificación del Banco Mundial. 
Respecto de la elasticidad ingreso, como es usual en la literatura, se encuentra que el gasto en salud funcionaría como un bien necesario (con un coeficiente de 0,742) mientras que entre regiones claramente aumenta a medida que aumentan las condiciones de desarrollo. Así, para las regiones de África, adopta valores de 0,5 a 0,65, mientras que los países desarrollados tienen una elasticidad que va de 0,16 a $0,8^{64} y$ América Latina arroja un valor de 0,66.

La última cuestión por evaluar es en qué medida este modelo es "explicativo" por regiones. Los aspectos vinculados con cada uno de los determinantes ya fueron evaluados previamente, por lo que solo resta evaluar la capacidad conjunta de explicar las variaciones del gasto. El análisis del $\mathrm{R}^{2}$ por regiones muestra que los mejores ajustes se dan para los países desarrollados $(0,92)$ seguidos por América Latina y Asia del Este con valores entre 0,85 y 0,88. Luego, ambas regiones de África se ubican en el rango de 0,75 y en último lugar el Sudeste Asiático con 0,4. Esta última región merece un comentario particular ya que, además de su baja capacidad explicativa global respecto del resto de las regiones, tanto el envejecimiento como el ingreso no parecen ser variables significativas, como sí la tecnología y la población en edad activa.

\begin{tabular}{|c|c|c|c|c|c|c|c|c|c|c|c|c|c|c|c|c|}
\hline & \multicolumn{2}{|c|}{ Basico } & \multicolumn{2}{|c|}{ Am. Lat. } & \multicolumn{2}{|c|}{ Am. Norte } & \multicolumn{2}{|l|}{ Europa } & \multicolumn{2}{|c|}{ Asia E. } & \multicolumn{2}{|c|}{ Asia $S$. } & \multicolumn{2}{|c|}{ N. Affica } & \multicolumn{2}{|c|}{ Africa SS. } \\
\hline & Base & $\begin{array}{l}\text { Edad } \\
\text { Activa }\end{array}$ & Base & $\begin{array}{l}\text { Edad } \\
\text { Activa }\end{array}$ & Base & $\begin{array}{l}\text { Edad } \\
\text { Activa }\end{array}$ & Base & $\begin{array}{l}\text { Edad } \\
\text { Activa }\end{array}$ & Base & $\begin{array}{l}\text { Edad } \\
\text { Activa }\end{array}$ & Base & $\begin{array}{l}\text { Edad } \\
\text { Activa }\end{array}$ & Base & $\begin{array}{l}\text { Edad } \\
\text { Activa }\end{array}$ & Base & $\begin{array}{l}\text { Edad } \\
\text { Activa }\end{array}$ \\
\hline \multirow[t]{2}{*}{ Envejecimienco } & 0.277 & 0.276 & 0.185 & 0.185 & .1216 & -0.390 & .0 .048 & 0.070 & 0.399 & 0.295 & 0.063 & 0.372 & 0.661 & 0.379 & 0.609 & 0.379 \\
\hline & {$[5,87)$} & $(5.75)$ & $(1,63)$ & $(1,60)$ & $(1773)$ & $(-1,77)$ & $(0,61)$ & $(0,81)$ & $(2,60)$ & (1.98\} & $(0,19)$ & (1,34) & (3.37) & (239) & $(2,63)$ & $(2,42)$ \\
\hline \multirow{2}{*}{ Ingreso } & 0.742 & 0.742 & 0,657 & 0,658 & 0.372 & 0.159 & 0,804 & & 0,768 & 0,330 & 0,026 & 0.253 & 0,498 & 0,498 & 0.640 & 0.651 \\
\hline & (31,0.64) & (31,04) & (11.0.04) & $(10,78)$ & 13.006 & $(1,52)$ & (22,561) & . & (13,16) & $(13,66)$ & (0.15) & $(1,75)$ & (9.92) & (592) & $(8,63)$ & $(8,7)$ \\
\hline \multirow[t]{2}{*}{ Tecnologia } & 39,56 & 39,36 & 48,83 & 48,30 & 101.9 & 91,16 & 45,07 & & 38,59 & 47,02 & 126,5 & 44,57 & 43,90 & 50.99 & 43,95 & 47,72 \\
\hline & (1481) & $(14.02)$ & (8.25) & (5s2) & (10.15) & $(11,33)$ & $(10.15)$ & 0 & (4.27) & (5.065) & (5.99) & (2.15) & (6994) & (5.44) & (5.93) & (6.19) \\
\hline \multirow[t]{2}{*}{ Edad Activa } & & $-0,019$ & & 0,032 & & 4,533 & & & & $-1,677$ & & 2.826 & & 0.459 & & -0.825 \\
\hline & & $(-0.14)$ & & $(0.08)$ & & $(4.48)$ & & 0 & & $(-3.23)$ & & (737) & & $\{-1.02\}$ & & $(-1,65)$ \\
\hline \multirow[t]{2}{*}{ Constance } & .301 .9 & .302 .8 & $.37,4$ & .367 .5 & $.66,4$ & .700 .5 & $.33+3.4$ & & $.295,2$ & .353 .6 & $-957,1$ & 348,2 & -332.6 & .384 .5 & .334 .9 & .360 .3 \\
\hline & (-13,0,0) & $(-14,3,1)$ & (.8334) & $(5,60)$ & +1025) & (-11,95j & $(-10.28)$ & 0 & $(-4,3)$ & 15,5089 & $(6,0,1)$ & (12224) & $(0,000)$ & $(5,549)$ & $(599)$ & $(-6,23)$ \\
\hline $\mathrm{R}^{2}$ & 0,920 & 0,920 & $\begin{array}{ll}0,987 \\
\end{array}$ & 0,087 & 0,39 & 0,109 & 0,929 & 0.926 & 0.848 & $0 ., 236$ & 0,161 & 0,411 & 0.734 & 0,754 & 0,774 & 0,788 \\
\hline Obserraciones & 2810 & 2810 & 479 & 479 & 32 & 32 & 768 & 768 & 398 & 398 & 126 & 126 & 282 & 282 & 725 & 725 \\
\hline
\end{tabular}

Fuente: Elaboración propia

Nota: Todos los modelos se estiman por paneles con efectos fijos.

Sobre la base de estos modelos se analiza la situación de América Latina, en especial con relación al incremento tendencial de costos en un contexto en el que la región se caracteriza por un proceso desigual de transición demográfica ${ }^{65}$, y en el que los gobiernos se comprometen a aumentos del gasto público en la búsqueda de mayores logros sanitarios removiendo barreras (financieras, de infraestructura, etc.) sobre el acceso $^{66}$. Es por esto que, en el contexto de las discusiones sobre protección social y salud, se realizan varios estudios sobre los efectos del envejecimiento ${ }^{67}$. Por eso, en la Tabla 4, primero se replican los resultados previos como punto de referencia. En la columna siguiente se trata de evaluar si es posible que también en la región exista un factor que lleve a una expansión creciente y sistemática del gasto. Curiosamente, cuando la tecnología se "desarma" en dos términos, uno lineal y otro cuadrático, ambos dejan de ser significativos. Nótese que este resultado sugiere que el efecto sería de tipo lineal, y por lo tanto no sería creciente, sólo positivo.

Luego, se realiza un ejercicio adicional para evaluar la "evolución" del modelo, intentando capturar la estabilidad temporal de los coeficientes. Para ello se realizan estimaciones por quinquenio, lo cual también implica que las estructuras de los datos convergen a ser de tipo cross-section, con lo cual los problemas comentados en la sección Antecedentes sobre este tipo de estimaciones también se aplican. De este estudio surge que el envejecimiento ha ido creciendo en importancia, al punto que en los primeros ańos no es estadísticamente significativo

${ }^{64}$ Este resultado debe tomarse con cuidado, dado que en América del Norte hay muy pocos países y con sistemas de salud muy diferentes entre ellos. ${ }^{65} \mathrm{Al}$ respecto puede verse Frenk et al (1991); o OPS (s/f); OPS (2017). ${ }^{66}$ OPS (2018).

${ }^{67}$ Solo a modo de ejemplo, para los países del Cono Sur: Rofman et al (2016) para Uruguay; Gragnolatti et al (2014) para la Argentina; Villalobos (2018) o Forascepi Crespo (2018) para Chile, pero también para países de América Central: como OPS (2013) para Mexico; o del Caribe: como Bayarre Vea et al (2018) para Cuba. 
mientras que en los últimos años lo es al 5\%. Por su parte, la proporción de población en edad activa pasa de ser significativa (al 10\%) a dejar de serlo, para luego volverse significativa nuevamente (también al 10\%). Un comportamiento similar presenta la elasticidad ingreso, aunque con un nivel de significatividad del $1 \%$ y siendo en el período intermedio casi del $10 \%$. En los períodos en que esta variable es significativa, supera el valor de uno ${ }^{68}$, por lo cual podría decirse que frente a la disponibilidad de nuevos recursos se produce una expansión y luego en algún momento se ajusta a la tendencia histórica. Finalmente, el efecto de la tecnología es creciente hasta 2010 para luego convertirse en no significativo e incluso pasar a tener signo negativo. Estos hechos sugieren que posiblemente la crisis financiera internacional de $2008^{69}$ haya tenido algún efecto sobre las finanzas públicas regionales.

Una especificación aún más flexible de la tecnología es la que se presenta en la última columna de la mencionada tabla, en la cual se incluye una dummy por año. En este caso, como es de esperarse, los valores convergen a los considerados como base (primera columna). Casi todos los años son significativos, a excepción de los primeros, lo cual posiblemente refleje que en un entorno de unos pocos ańos el gasto no cambia significativamente ${ }^{70}$. Además, los valores de los coeficientes muestran que este alejamiento (haciendo referencia al incremento absoluto y relativo) en realidad llega a su valor máximo (por encima del 25\%) en 2008-2009 y luego se comporta de forma estable por debajo del 10\%.

Tabla 4. La tecnología en América Latina

\begin{tabular}{|c|c|c|c|c|c|c|}
\hline & Base & Tec. cuad. & 2000-2005 & 2005-2010 & 2010-2015 & Tec. discreta \\
\hline \multirow[t]{2}{*}{ Envejecimiento } & 0,185 & 0,194 & $-0,269$ & 0,520 & 0,655 & 0,183 \\
\hline & $(1,60)$ & $(1,67)$ & $(-0,79)$ & $(1,45)$ & $(2,01)$ & $(1,56)$ \\
\hline \multirow[t]{2}{*}{ Ingreso } & 0,658 & 0,646 & 1,120 & 0,216 & 1,149 & 0,672 \\
\hline & $(10,78)$ & $(10,25)$ & $(7,22)$ & $(1,65)$ & $(6,63)$ & $(10,01)$ \\
\hline \multirow[t]{2}{*}{ Tecnología } & 48,30 & 14390,1 & 53,28 & 119,9 & $-9,433$ & \\
\hline & $(5,52)$ & $(0,80)$ & $(2,56)$ & $(4,51)$ & $(-0,44)$ & \\
\hline Edad Activa & 0,032 & $-0,017$ & $-1,952$ & $-1,003$ & $-1,646$ & $-0,014$ \\
\hline \multirow[t]{2}{*}{$(\text { Tecnologia })^{2}$} & & $-942,9$ & & & & \\
\hline & & $(-0,80)$ & & & & \\
\hline \multirow[t]{2}{*}{ Añ́o $=2000$} & & & & & & 0 \\
\hline & & & & & & (.) \\
\hline \multirow[t]{2}{*}{ Año $=2001$} & & & & & & 0,032 \\
\hline & & & & & & $(1,14)$ \\
\hline \multirow[t]{2}{*}{ Año $=2002$} & & & & & & 0,052 \\
\hline & & & & & & $(1,81)$ \\
\hline \multirow[t]{2}{*}{ Ańo $=2003$} & & & & & & 0,086 \\
\hline & & & & & & $(2,86)$ \\
\hline \multirow[t]{2}{*}{ Año $=2004$} & & & & & & 0,090 \\
\hline & & & & & & $(2,79)$ \\
\hline \multirow[t]{2}{*}{ Año $=2005$} & & & & & & 0,105 \\
\hline & & & & & & $(2,94)$ \\
\hline \multirow{2}{*}{ Año $=2006$} & & & & & & 0,115 \\
\hline & & & & & & $(2,87)$ \\
\hline \multirow[t]{2}{*}{ Año $=2007$} & & & & & & 0,156 \\
\hline & & & & & & $(3,52)$ \\
\hline \multirow[t]{2}{*}{ Ańo $=2008$} & & & & & & 0,193 \\
\hline & & & & & & $(4,03)$ \\
\hline \multirow[t]{2}{*}{ Ańo $=2009$} & & & & & & 0,259 \\
\hline & & & & & & $(5,25)$ \\
\hline \multirow[t]{2}{*}{ Añ́o $=2010$} & & & & & & 0,266 \\
\hline & & & & & & $(5,05)$ \\
\hline \multirow[t]{2}{*}{ Ańo $=2011$} & & & & & & 0,281 \\
\hline & & & & & & $(4,97)$ \\
\hline \multirow[t]{2}{*}{ Ańo $=2012$} & & & & & & 0,296 \\
\hline & & & & & & $(4,92)$ \\
\hline \multirow[t]{2}{*}{ Ańo $=2013$} & & & & & & 0.308 \\
\hline & & & & & & $(4,84)$ \\
\hline \multirow[t]{2}{*}{ Año $=2014$} & & & & & & 0,317 \\
\hline & & & & & & $(4,73)$ \\
\hline \multirow[t]{2}{*}{ Ańo $=2015$} & & & & & & 0,343 \\
\hline & & & & & & $(4,89)$ \\
\hline \multirow[t]{2}{*}{ Constante } & $-367,5$ & $-54904,5$ & $-400,5$ & $-904,4$ & 73,21 & $-0,304$ \\
\hline & $(-5,66)$ & $(-0,81)$ & $(-2,59)$ & $(-4,59)$ & $(0,46)$ & $(-0,16)$ \\
\hline $\mathrm{R}^{2}$ & 0,887 & 0,886 & 0,742 & 0,667 & 0,903 & 0,889 \\
\hline Observaciones & 479 & 479 & 150 & 150 & 179 & 479 \\
\hline
\end{tabular}

Fuente: Elaboración propia

Nota: Todos los modelos se estiman por paneles con efectos fijos.

${ }^{68} \mathrm{Al}$ segmentar por quinquenio los datos se acercan a ser de tipo cross-section, por lo cual debe recordarse que en la revisión de la literatura se comentó que los primeros estudios utilizaban este tipo de estructuras y los resultados eran similares a los obtenidos.

${ }^{69}$ Como lo sugiere OPS (2018).

${ }^{70}$ Daría la idea de cierta rigidez, y por lo tanto sugiere que la hipótesis de paneles dinámicos debería ser explorada. 
Finalmente, resulta interesante saber cuál es la contribución de cada factor a los cambios en el gasto ${ }^{71}$, resultados que se presentan en la Tabla 5. Allí vemos que, en promedio, el principal impulsor del gasto es el ingreso, que contribuye con el 51,8\% de las variaciones, mientras que el envejecimiento resulta otro factor significativo, con una contribución del 30,6\%, quedando para la tecnología el 17,5\% restante. Cuando este análisis se realiza por determinante resulta que, en cuanto al envejecimiento, la contribución es mayor en el Sudeste asiático, seguido por Europa y Asia Central, ambas por encima del 40\%; luego, en el Este asiático, América del Norte y América Latina, en el orden del 35\%, mientras que en África se ubica por debajo del $20 \%$. Si se evalúa la situación del ingreso, surge que es el principal factor en África del Norte, con 65\% de los cambios del gasto por persona, seguida por América del Norte, con el 58\%. La región en la que menos contribuye es Asia del Sur con el 38,7\%. Finalmente, la principal contribución de la tecnología se presenta en África sub sahariana con un 32,7\%, mientras que en el resto de las regiones se ubica en torno o por debajo del promedio general. Es en los países desarrollados (América del Norte y Europa) en donde se observa la menor contribución, del orden del 5\%.

Tabla 5. Contribución de cada determinante al cambio del Gasto en Salud

\begin{tabular}{lccc} 
& & Determinante & Tecnología \\
\hline Asia E. & Envejecimiento & Ingreso & 18,19 \\
Europa y Asia Central & 37,51 & 44,30 & 5,79 \\
América Latina y Caribe & 41,49 & 52,72 & 15,69 \\
África N. & 38,07 & 46,24 & 15,81 \\
Asia S. & 19,43 & 64,77 & 17,65 \\
África SS. & 43,63 & 38,72 & 32,72 \\
Total & 11,48 & 55,80 & 17,54 \\
\hline Observaciones & 30,64 & 51,82 & \\
\hline
\end{tabular}

Fuente: Sobre la base de las estimaciones del modelo "Activa" de la Tabla 3

\section{IV.2. CONTROLANDO POR LA ORGANIZACIÓN DEL SISTEMA DE SALUD}

Una dificultad clave en las estimaciones anteriores es la comparación, y la intuición subyacente, de resultados para sistemas de salud que tienen organizaciones y respuestas de los hacedores de política peculiares o propias. Si bien la metodología econométrica utilizada permite controlar por estos aspectos (motivación principal para estimar modelos de datos en panel con efectos fijos ${ }^{72}$ ) al no poder establecer cuáles son las particularidades resulta difícil comprender mejor los resultados. Adicionalmente, tampoco es posible controlar qué parte de los efectos que computa la variable tecnología se deben a ella misma y qué parte a las cuestiones relacionadas con la enfermedad de costos. Ambos componentes tienen tendencia creciente, pero claramente las medidas de política pública son bien distintas en cada caso.

Una segunda cuestión que resulta difícil de computar utilizando grandes paneles de países es la hipótesis de envejecimiento versus momento de la muerte. En ambos casos, si se pudiese disponer de microdatos sobre condiciones socioeconómicas, podrían computarse indicadores para testear estas hipótesis. Por eso se plantea realizar un estudio a nivel de un único país (Argentina) con un sistema público relativamente homogéneo en cuanto a su organización, tanto en las condiciones de acceso como en las de mercados de insumos que enfrenta el Estado ${ }^{73}$. En este contexto, debe quedar en claro que no se trata de un modelo

${ }^{71}$ Estas estimaciones, siguiendo a Di Matteo (2005), surgen de comparar los resultados al inicio y al final de los valores predichos por los modelos. ${ }^{72} \mathrm{Del}$ mismo modo que para el análisis a nivel país, la intuición sugiere realizar la estimación del modelo para las provincias argentinas mediante efectos fijos, con el fin de capturar sus diferencias idiosincráticas. Aun así, se realizó un Test de Hausman (1978) para el modelo básico, el cual arrojó una Prob>chi²=0,005. Este resultado respalda la elección respecto al método de estimación utilizado.

${ }^{73}$ Principalmente respecto del insumo más importante: los RRHH. 
de sistema único, y por eso precisamente resulta interesante, sino que los mercados de factores tienen una comunicación y una institucionalidad más homogénea en términos relativos a los estudios previos, de múltiples casos con estructuras institucionales y económicas bien diferentes.

Este estudio se realiza en tres conjuntos de modelos, todos los cuales se caracterizan por coeficientes de ajustes globales incluso más elevados a los analizados previamente, con $\mathrm{R}^{2}$ que llegan casi al $95 \%$. En el primero, Tabla 6 , se trata de testear si el modelo general ajusta de manera similar a lo que sucede con el resto del mundo, y luego si variaciones en este inciden sobre los resultados. La principal conclusión es que el modelo general se sigue validando en el contexto de América Latina, esto es: el envejecimiento no es significativo, sí lo es el ingreso (aunque con valores inferiores, del orden del 0,55) y la tecnología también es relevante. La incorporación de la población sin seguro, posiblemente de manera contra-intuitiva, no resulta significativa ${ }^{74}$. Con respecto a la enfermedad de costos, sólo resulta significativo el índice sobre la base de salario horario.

Tabla 6. Modelos básicos

\begin{tabular}{|c|c|c|c|c|c|}
\hline & Base & Edad activa & Sin seguro & $\begin{array}{l}\text { Enf. costos } \\
\text { ingresos }\end{array}$ & $\begin{array}{l}\text { Enf. costos } \\
\text { salario } h \text {. }\end{array}$ \\
\hline \multirow[t]{2}{*}{ Envejecimiento } & 0,139 & 0,196 & 0,198 & 0,186 & 0,168 \\
\hline & $(0,91)$ & $(1,27)$ & $(1,25)$ & $(1,18)$ & $(1,05)$ \\
\hline \multirow[t]{2}{*}{ Ingreso } & 0,587 & 0,545 & 0,549 & 0,537 & 0,551 \\
\hline & $(5,70)$ & $(5,23)$ & $(4,88)$ & $(4,77)$ & $(4,92)$ \\
\hline \multirow[t]{2}{*}{ Tecnología } & 147,8 & 152,8 & 152,3 & 156,8 & 153,7 \\
\hline & $(5,38)$ & $(5,57)$ & $(5,41)$ & $(5,54)$ & $(5,49)$ \\
\hline \multirow[t]{2}{*}{ Edad Activa } & & 1,227 & 1,227 & 1,261 & 1,224 \\
\hline & & $(2,10)$ & $(2,10)$ & $(2,15)$ & $(2,10)$ \\
\hline \multirow{2}{*}{ Población sin seg. salud } & & & 0,007 & 0,008 & 0,007 \\
\hline & & & $(0,08)$ & $(0,10)$ & $(0,08)$ \\
\hline \multirow[t]{2}{*}{ Enf. de costos ing. mensual } & & & & 0,101 & \\
\hline & & & & $(1,34)$ & \\
\hline \multirow[t]{2}{*}{ Enf. de costos salario horario } & & & & & 0,124 \\
\hline & & & & & $(1,99)$ \\
\hline \multirow[t]{2}{*}{ Constante } & $-1121,4$ & $-1164,0$ & $-1160,3$ & $-1195,1$ & $-1171,5$ \\
\hline & $(-5,38)$ & $(-5,60)$ & $(-5,44)$ & $(-5,57)$ & $(-5,52)$ \\
\hline $\mathrm{R}^{2}$ & 0,943 & 0,944 & 0,944 & 0,944 & 0,945 \\
\hline Observaciones & 299 & 299 & 299 & 299 & 299 \\
\hline
\end{tabular}

Fuente: Elaboración propia sobre la base de datos de la Secretaría de Hacienda de la Nación y SEDLAC (CEDLAS y The World Bank) Nota: Todos los modelos se estiman por paneles con efectos fijos.

Estos modelos son luego modificados y presentados en la Tabla 7 para incluir el efecto de la tecnología pero como dummies por año. Los dos principales cambios que se perciben son: que la elasticidad ingreso aumenta en valor, llegando casi al 0,9, mientras que el efecto de la enfermedad de costos, que antes era significativo, ahora se diluye. Es interesante observar la evolución de los coeficientes de las dummies anuales dado que, como veíamos para el caso de América Latina en la Tabla 4, muestran que en el año previo a la crisis de 2008 se produce un salto discreto muy significativo en los niveles de gasto, para luego seguir la tendencia ascendente previa.

${ }^{74}$ Se esperaría que esta variable sea significativa debido a que es un resultado establecido y conocido que los usuarios del sistema público de salud son, principalmente, personas en situación de pobreza que a no pueden disponer de un seguro de salud, ya sea por falta de ingresos o por trabajos informales. En Crosta (2009) se puede ver una revisión de estos resultados, a la par de una descomposición que permite establecer las causas de esta focalización 
Tabla 7. Modelos con tendencia flexible

\begin{tabular}{|c|c|c|c|c|c|}
\hline & Base & Edad activa & Sin seguro & $\begin{array}{l}\text { Enf. costos } \\
\text { ingresos }\end{array}$ & $\begin{array}{l}\text { Enf. costos } \\
\text { salario h. }\end{array}$ \\
\hline \multirow[t]{2}{*}{ Envejecimiento } & $-0,034$ & 0,011 & 0,039 & 0,033 & 0,017 \\
\hline & $(-0,22)$ & $(0,07)$ & $(0,25)$ & $(0,21)$ & $(0,11)$ \\
\hline \multirow[t]{2}{*}{ Ingreso } & 0,852 & 0,824 & 0,887 & 0,864 & 0,882 \\
\hline & $(6,18)$ & $(5,96)$ & $(5,96)$ & $(5,76)$ & $(5,93)$ \\
\hline \multirow[t]{2}{*}{ Año $=2003$} & 0 & 0 & 0 & 0 & 0 \\
\hline & (,) & $()$, & (,) & $()$, & $()$, \\
\hline \multirow[t]{2}{*}{ Año $=2004$} & 0,067 & 0,066 & 0,065 & 0,063 & 0,064 \\
\hline & $(1,55)$ & $(1,52)$ & $(1,50)$ & $(1,46)$ & $(1,48)$ \\
\hline \multirow[t]{2}{*}{ Año $=2005$} & 0,147 & 0,144 & 0,140 & 0,143 & 0,141 \\
\hline & $(2,96)$ & $(2,90)$ & $(2,83)$ & $(2,89)$ & $(2,85)$ \\
\hline \multirow[t]{2}{*}{ Año $=2006$} & 0,133 & 0,129 & 0,119 & 0,131 & 0,123 \\
\hline & $(2,02)$ & $(1,96)$ & $(1,79)$ & $(1,96)$ & $(1,86)$ \\
\hline \multirow[t]{2}{*}{ Año = 2007} & 0,333 & 0,329 & 0,319 & 0,330 & 0,316 \\
\hline & $(4,84)$ & $(4,79)$ & $(4,60)$ & $(4,72)$ & $(4,57)$ \\
\hline \multirow[t]{2}{*}{ Año $=2008$} & 0,280 & 0,278 & 0,257 & 0,276 & 0,263 \\
\hline & $(2,60)$ & $(2,59)$ & $(2,37)$ & $(2,52)$ & $(2,43)$ \\
\hline \multirow[t]{2}{*}{ Año = 2009} & 0,288 & 0,286 & 0,258 & 0,280 & 0,260 \\
\hline & $(2,35)$ & $(2,35)$ & $(2,07)$ & $(2,23)$ & $(2,09)$ \\
\hline \multirow[t]{2}{*}{ Año = 2010} & 0,287 & 0,291 & 0,256 & 0,281 & 0,264 \\
\hline & $(2,16)$ & $(2,19)$ & $(1,88)$ & $(2,04)$ & $(1,95)$ \\
\hline \multirow[t]{2}{*}{ Año = 2011} & 0,343 & 0,347 & 0,306 & 0,336 & 0,317 \\
\hline & $(2,23)$ & $(2,26)$ & $(1,94)$ & $(2,11)$ & $(2,02)$ \\
\hline \multirow[t]{2}{*}{ Año $=2012$} & 0,392 & 0,395 & 0,348 & 0,378 & 0,356 \\
\hline & $(2,38)$ & $(2,41)$ & $(2,06)$ & $(2,21)$ & $(2,11)$ \\
\hline \multirow[t]{2}{*}{ Año $=2013$} & 0,426 & 0,432 & 0,380 & 0,413 & 0,390 \\
\hline & $(2,32)$ & $(2,36)$ & $(2,01)$ & $(2,17)$ & $(2,07)$ \\
\hline \multirow[t]{2}{*}{ Año $=2014$} & 0,424 & 0,437 & 0,384 & 0,421 & 0,398 \\
\hline & $(2,20)$ & $(2,27)$ & $(1,94)$ & $(2,11)$ & $(2,02)$ \\
\hline \multirow[t]{2}{*}{ Año $=2015$} & 0,644 & 0,656 & 0,598 & 0,638 & 0,613 \\
\hline & $(3,19)$ & $(3,26)$ & $(2,88)$ & $(3,04)$ & $(2,96)$ \\
\hline \multirow[t]{2}{*}{ Edad Activa } & & 0,965 & 0,949 & 0,971 & 0,966 \\
\hline & & $(1,70)$ & $(1,67)$ & $(1,71)$ & $(1,70)$ \\
\hline \multirow[t]{2}{*}{ Población sin seg. salud } & & & 0,098 & 0,098 & 0,096 \\
\hline & & & $(1,13)$ & $(1,14)$ & $(1,11)$ \\
\hline \multirow[t]{2}{*}{ Enf. de costos ing. mensual } & & & & 0,087 & \\
\hline & & & & $(1,18)$ & \\
\hline \multirow[t]{2}{*}{ Enf. de costos salario horario } & & & & & 0,095 \\
\hline & & & & & $(1,57)$ \\
\hline \multirow[t]{2}{*}{ Constante } & 1,000 & $-2,834$ & $-3,553$ & $-3,528$ & $-3,563$ \\
\hline & $(1,32)$ & $(-1,19)$ & $(--1,44)$ & $(-1,43)$ & $(-1,45)$ \\
\hline $\mathrm{R}^{2}$ & 0,950 & 0,951 & 0,951 & 0,951 & 0,952 \\
\hline Observaciones & 299 & 299 & 299 & 299 & 299 \\
\hline
\end{tabular}

Fuente: Elaboración propia sobre la base de datos de la Secretaría de Hacienda de la Nación y SEDLAC (CEDLAS y The World Bank) Nota: Todos los modelos se estiman por paneles con efectos fijos.

Finalmente, en la Tabla 8, se presentan los resultados producto de incluir la estructura de edades de la población mayor a 60 años, con el objetivo de tratar de probar la hipótesis de envejecimiento versus momento de la muerte. Lo que se observa es que el coeficiente de la población mayor a los 80 años es significativo, lo cual podría estar reflejando que el efecto significativo refiere efectivamente a la hipótesis de cercanía a la muerte más que a la de vejez. Obviamente esta hipótesis requiere de una evaluación más intensa, aunque de difícil realización para un país que no dispone de un sistema de historias clínicas unificado. 
Tabla 8. Modelos con edad flexible y enfermedad de costos

\begin{tabular}{|c|c|c|c|c|c|}
\hline & Base & Edad activa & Sin seguro & $\begin{array}{l}\text { Enf. costos } \\
\text { ingresos }\end{array}$ & $\begin{array}{l}\text { Enf. costos salario } \\
\text { h. }\end{array}$ \\
\hline \multirow[t]{2}{*}{ Ingreso } & 0,794 & 0,773 & 0,851 & 0,821 & 0,844 \\
\hline & $(5,69)$ & $(5,54)$ & $(5,71)$ & $(5,46)$ & $(5,67)$ \\
\hline \multirow[t]{2}{*}{ Envejecimiento 61-70 } & $-0,076$ & $-0,045$ & $-0,030$ & $-0,040$ & $-0,046$ \\
\hline & $(-0,78)$ & $(-0,46)$ & $(-0,30)$ & $(-0,40)$ & $(-0,46)$ \\
\hline \multirow[t]{2}{*}{ Envejecimiento $71-80$} & 0,060 & 0,062 & 0,077 & 0,084 & 0,080 \\
\hline & $(0,73)$ & $(0,76)$ & $(0,94)$ & $(1,03)$ & $(0,98)$ \\
\hline \multirow[t]{2}{*}{ Envejecimiento +80} & 0,096 & 0,098 & 0,111 & 0,113 & 0,104 \\
\hline & $(1,85)$ & $(1,88)$ & $(2,11)$ & $(2,16)$ & $(1,97)$ \\
\hline \multirow[t]{2}{*}{ Ańo $=2003$} & 0 & 0 & 0 & 0 & 0 \\
\hline & (,) & (,) & (,) & (,) & (,) \\
\hline \multirow[t]{2}{*}{ Año $=2004$} & 0,080 & 0,079 & 0,078 & 0,076 & 0,077 \\
\hline & $(1,84)$ & $(1,80)$ & $(1,80)$ & $(1,76)$ & $(1,77)$ \\
\hline \multirow[t]{2}{*}{ Año $=2005$} & 0,161 & 0,156 & 0,153 & 0,157 & 0,154 \\
\hline & $(3,22)$ & $(3,14)$ & $(3,06)$ & $(3,16)$ & $(3,10)$ \\
\hline \multirow[t]{2}{*}{ Año $=2006$} & 0,156 & 0,150 & 0,139 & 0,155 & 0,144 \\
\hline & $(2,35)$ & $(2,26)$ & $(2,08)$ & $(2,30)$ & $(2,16)$ \\
\hline \multirow[t]{2}{*}{ Año $=2007$} & 0,355 & 0,349 & 0,338 & 0,352 & 0,335 \\
\hline & $(5,12)$ & $(5,04)$ & $(4,85)$ & $(5,01)$ & $(4,83)$ \\
\hline \multirow[t]{2}{*}{ Ańo $=2008$} & 0,322 & 0,317 & 0,293 & 0,318 & 0,300 \\
\hline & $(2,96)$ & $(2,92)$ & $(2,69)$ & $(2,88)$ & $(2,75)$ \\
\hline \multirow[t]{2}{*}{ Ańo $=2009$} & 0,339 & 0,333 & 0,300 & 0,329 & 0,303 \\
\hline & $(2,74)$ & $(2,70)$ & $(2,39)$ & $(2,59)$ & $(2,43)$ \\
\hline \multirow[t]{2}{*}{ Año $=2010$} & 0,337 & 0,336 & 0,294 & 0,327 & 0,305 \\
\hline & $(2,50)$ & $(2,50)$ & $(2,15)$ & $(2,36)$ & $(2,23)$ \\
\hline \multirow[t]{2}{*}{ Ańo $=2011$} & 0,403 & 0,401 & 0,352 & 0,391 & 0,366 \\
\hline & $(2,58)$ & $(2,57)$ & $(2,21)$ & $(2,42)$ & $(2,30)$ \\
\hline \multirow[t]{2}{*}{ Ańo $=2012$} & 0,449 & 0,447 & 0,390 & 0,430 & 0,401 \\
\hline & $(2,70)$ & $(2,69)$ & $(2,28)$ & $(2,49)$ & $(2,35)$ \\
\hline \multirow{2}{*}{ Ańo $=2013$} & 0,490 & 0,489 & 0,427 & 0,471 & 0,440 \\
\hline & $(2,63)$ & $(2,63)$ & $(2,24)$ & $(2,45)$ & $(2,32)$ \\
\hline \multirow[t]{2}{*}{ Año $=2014$} & 0,499 & 0,503 & 0,440 & 0,489 & 0,458 \\
\hline & $(2,55)$ & $(2,57)$ & $(2,21)$ & $(2,42)$ & $(2,30)$ \\
\hline \multirow[t]{2}{*}{ Ańo $=2015$} & 0,723 & 0,727 & 0,658 & 0,710 & 0,675 \\
\hline & $(3,53)$ & $(3,55)$ & $(3,14)$ & $(3,34)$ & $(3,23)$ \\
\hline \multirow[t]{2}{*}{ Edad Activa } & & 0,867 & 0,838 & 0,854 & 0,851 \\
\hline & & $(1,52)$ & $(1,47)$ & $(1,50)$ & $(1,50)$ \\
\hline \multirow[t]{2}{*}{ Población sin seg. salud } & & & 0,127 & 0,129 & 0,125 \\
\hline & & & $(1,47)$ & $(1,49)$ & $(1,44)$ \\
\hline \multirow[t]{2}{*}{ Enf. de costos ing. mensual } & & & & 0,102 & \\
\hline & & & & $(1,39)$ & \\
\hline \multirow[t]{2}{*}{ Enf. de costos salario horario } & & & & & 0,092 \\
\hline & & & & & $(1,52)$ \\
\hline \multirow[t]{2}{*}{ Constante } & 1,289 & $-2,144$ & $-2,983$ & $-2,909$ & $-2,987$ \\
\hline & $(1,69)$ & $(-0,90)$ & $(-1,22)$ & $(-1,19)$ & $(-1,22)$ \\
\hline $\mathrm{R}^{2}$ & 0,951 & 0,952 & 0,952 & 0,952 & 0,953 \\
\hline Observaciones & 299 & 299 & 299 & 299 & 299 \\
\hline
\end{tabular}

Fuente: Elaboración propia sobre la base de datos de la Secretaría de Hacienda de la Nación y SEDLAC (CEDLAS y The World Bank) Nota: Todos los modelos se estiman por paneles con efectos fijos.

Al evaluar la contribución de cada factor al cambio en el gasto, Tabla 9, surgen cambios notables respecto de los casos globales. La primera diferencia es que la tecnología contribuye con algo más de la mitad del cambio, mientras que el ingreso es el responsable del $45 \%$, por lo que entre ambas variables prácticamente se podría explicar la totalidad de la variación, quedando para el envejecimiento una contribución levemente superior al 3\%. Esta menor relevancia de las variables que identifican envejecimiento, podría ser otra forma de denotar la ausencia de significatividad de la proporción de población sin seguro, beneficiarios en gran medida de este gasto, los cuales suelen ser individuos de bajos ingresos y con una menor edad promedio que el conjunto de la sociedad. 
Tabla 9. Contribución de cada determinante al cambio del Gasto en Salud

\begin{tabular}{lc} 
Envejecimiento & $\begin{array}{c}\text { Contribuciones } \\
\text { (en porcentaje) }\end{array}$ \\
Edad activa & 2,54 \\
Ingreso & 1,24 \\
Tecnología & 45,24 \\
\hline
\end{tabular}

Fuente: Estimación propia sobre la base de las estimaciones del modelo "Activa" de la Tabla 6

\section{CONCLUSIONES}

El nivel y la tendencia del gasto público global en salud y de las regiones/países se encuentra en el centro de la agenda de estudio en el sector salud. Sucede que existe un conjunto de países que parten de un nivel de salud más que aceptable (los desarrollados) y presentan una tendencia de crecimiento permanente del gasto en salud, mientras que otro conjunto de países que deberían aumentarlo (debido al precario nivel de salud de su población) enfrentan mayores dificultades presupuestarias e institucionales para lograrlo de manera consistente y efectiva.

Este documento trata de analizar un modelo comúnmente aceptado sobre el gasto público en salud, que sostiene su determinación a través de tres variables clave: el envejecimiento, los ingresos y la tecnología. La principal conclusión a la que se arriba es que, a través de un análisis empírico de modelos lineales para datos en panel con efectos fijos, esta idea se convalida en términos generales (salvo para el Sudeste Asiático). Claramente, comprender y estudiar este resultado conlleva un nivel de análisis que excede el objetivo del presente trabajo.

Con respecto al primero de los determinantes, el envejecimiento, se afirma que un cambio en la estructura demográfica (en las tasas de natalidad y mortalidad) se presupone demandante neto de recursos en el largo plazo. En este trabajo se encuentra que, como se esperaría, la estructura poblacional condiciona los cambios en el gasto, pero lo hace de manera suave. No obstante, entre regiones se puede ver que el efecto resulta muy significativo para Asia y África, pero no para el resto del mundo, en donde el aumento de la población en edad activa resulta significativo. De hecho, su contribución a los cambios en el gasto por persona es, en promedio, de un 30\%, pero en algunas regiones apenas supera el 11\%. Pese a estos resultados, al estudiar el caso particular de la República Argentina surge que esta variable es poco significativa, siendo relevante la población económicamente activa (posiblemente por el estado demográfico del país). Una posible extensión de este trabajo consistiría en incluir una variable que capture, entre países o provincias, las diferencias en la transición demográfica. Finalmente, el ejercicio para Argentina también permite corroborar, al menos parcialmente, la idea de que el gasto no cambia por el envejecimiento sino por la probabilidad de muerte. Es así que, cuando se separa la población mayor de 60 años en tres grupos (por decenios) sólo el último, en donde claramente la probabilidad de muerte tiende a elevarse de forma significativa por superar el valor de la esperanza de vida, resulta ser significativo. De esta manera, un estudio más detallado de este punto podría resultar en un mejor entendimiento de la dinámica de vejez, muerte, hospitalización y gasto.

En cuanto a los ingresos, los resultados obtenidos se encuentran bastante alineados con aquello que podría esperarse por la revisión de la literatura, surgiendo que explican alrededor de la mitad de las variaciones del gasto público por persona. En este sentido, los resultados indican que el gasto en salud se comporta como un bien necesario y que, además, su elasticidad es variable siendo creciente, sin llegar a uno, a medida que las regiones se desarrollan. Incluso, el análisis específico para América Latina indica que (replicando a los primeros estudios de cross-section) cuando el ingreso aumenta también lo hace el gasto, pero cuando 
el primero se retrotrae o aumenta poco, el gasto se comporta de forma similar. Así, en el período de la crisis de 2008 la elasticidad ingreso es muy baja (un tercio de los valores esperados), pero antes y después de la misma se eleva por encima de 1. Esta hipótesis podría evaluarse en un estudio posterior mediante la inclusión de una variable dummy que refleje la interacción de los ingresos con la crisis financiera de 2008.

Finalmente, la tecnología, entendida como la presión exógena y permanente del gasto al alza, es significativa tanto en términos globales como por regiones, lo cual sugiere que la afirmación sobre que el gasto tiene una tendencia al alza permanente podría ser cierta. Si se consideran las especificaciones con variables dummies por año, para América Latina y la Argentina es en el año previo a la crisis de 2008 en donde comienza un proceso distinto de gasto: básicamente un escalón más arriba y con la misma tendencia ascendente. Este resultado es importante, ya que sugiere que la tendencia del gasto no sería explosiva. La contribución de esta variable es del 18\%, en promedio, aunque en algunas regiones aporta más (como en África subsahariana), lo cual podría deberse a la necesidad de este grupo de países de converger al nivel de gasto internacionales. En el caso puntual de la Argentina, esta variable captura casi la mitad del cambio en el gasto.

El estudio específico de un país permite analizar un segundo canal por el cual la tecnología afectaría al gasto: la "Enfermedad de Costos" de Baumol. En este caso, el concepto refiere a la forma de la función de producción que, al ser dependiente del capital humano, deriva en la necesidad de aumentos salariales por encima del promedio de la economía. Como se dispone de microdatos de una encuesta destinada a estudiar el mercado laboral, es posible construir dos medidas a partir de los ingresos laborales: una de tipo mensual y otra horaria. Como sería de esperar por su mayor cercanía al concepto de productividad, la segunda es la única que resulta significativa, aunque muy débilmente. Este resultado es importante porque, de los cuatro determinantes aquí considerados, es la enfermedad de costos (por su complejidad más allá de lo económico y su efecto directo sobre los costos) una dimensión que requiere un foco especial de atención por parte de los responsables de las políticas sectoriales.

En síntesis, en este documento se realiza un estudio de un modelo comúnmente aceptado de determinación del gasto público en salud. Del mismo surge que el ingreso y el envejecimiento son variables claves en su evolución, a la par que se identifica una tendencia exógena positiva, que aquí se denomina tecnología. Este modelo se aplica a distintos niveles geográficos: el mundo, regiones, países, estrategia que demuestra su utilidad al permitir controlar por efectos no observables asociados a los diferentes modos de organización de los sistemas de salud, pero también a las distintas respuestas que pueden dar los países en términos fiscales.

\section{REFERENCIAS}

Aprile R. (2007). How to Take Account of Death-Related Costs in Projecting Health Care Expenditure Updated Version. Ragioneria Generale Dello Stato, Italy.

Astolfi R., Lorenzoni L. y Oderkirk J. (2012). A Comparative Analysis of Health Forecasting Methods. Health Working Papers N 59. OECD Publishing Paris http://dx.doi.org/10.1787/5k912j389 en.

Baltagi B. H., Lagravinese R., Moscone F. y Tosetti E. (2017). Health Care Expenditure and Income: A Global Perspective. Health Economics, 26 (7), 863-874.

Baltagi, B. H. y Moscone, F. (2010). Health Care Expenditure and Income in the OECD Reconsidered: Evidence from Panel Data. Economic Modelling, 27, 804-811. http://dx. doi.org/10.1016/j.econ$\bmod .2009 .12 .001$ 
Bates, L. J. y Santerre, R. (2013). Does the U.S. health care sector suffer from Baumol's cost disease? Evidence from the 50 states. Journal of Health Economics, 32 (2), 386-391.

Baumol W. J. (1967). Macroeconomics of Unbalanced Growth: The Anatomy of Urban Crisis. The American Economic Review, 57(3), 415-426.

Baumol, W. J. (2012). The cost disease: why computers get cheaper and health care doesn't. Yale University Press.

Bayarre Vea H. D., Álvarez Lauzarique M. E., Pérez Piñero J. S., Almenares Rodríguez K., Rodríguez Cabrera A., Pría Barros M. C., et al. (2018). Enfoques, evolución y afrontamiento del envejecimiento demográfico en Cuba. Rev. Panam. Salud Publica, 42, e21. https://doi.org/10.26633/RPSP.2018.21

Breyer, F. y Felder, F. (2006). Life expectancy and health care expenditure: a new calculation for Germany using the costs of dying. Health Policy, 75(2), 178-186.

Cetrángolo, O., Gómez Sabaini, J. C. y Morán, D. (2015). Argentina: reformas fiscales, crecimiento e inversión (2000-2014). Macroeconomía del Desarrollo 165, Naciones Unidas Comisión Económica para América Latina y el Caribe.

Chandra, A. y Skinner, J. (2012). Technology Growth and Expenditure Growth in Health Care. Journal of Economic Literature, 50 (3), 645-80. doi: 10.1257/jel.50.3.645

Chernew M. E. y Newhouse J. P. (2012). Health Care Spending Growth. In: Handbook of Health Economics. Volume 2. Elsevier B.V.

Colombier, C. (2012). Drivers of health care expenditure: does Baumol's cost disease loom large?. FiFo Discussion Papers 12(5).

Crosta, F. L. (2009). Caracterización de cambios en el uso de servicios de salud con microdesomposiciones. Anales de Asociación Argentina de Economía Política ISBN 978-987-99570-7-3, ISSN 1852-0022

Crosta, F. L. (2018). Análisis de la evolución del Gasto Público en Salud en Argentina 2000-2015, proyecciones y determinantes. Anales Jornadas Internacionales Economía de la Salud AES -SAP

Culyer, A. J. (2016). Cost-effectiveness thresholds in health care: a bookshelf guide to their meaning and use. Health Economics, Policy and Law, 11, 415-432. doi:10.1017/S1744133116000049

De la Maisonneuve, C. y Oliveira Martins, J. (2013). A Projection Method for Public Health and LongTerm Care Expenditures. OECD Economics Department Working Papers, No. 1048, OECD Publishing. http://dx.doi.org/10.1787/5k44v53w5w47-en.

De la Maisonneuve, C., Moreno-Serra, R., Murtin, F., y Oliveira Martins, J. (2016). The Role of Policy and Institutions on Health Spending. Health Economics, 26(7), 834-843. doi:10.1002/hec.3410

De Meijer, C., Wouterse, B., Polder, J., y Koopmanschap, M. (2013). The effect of population aging on health expenditure growth: a critical review. European journal of ageing, 10(4), 353-361. doi:10.1007/ s10433-013-0280-x 
Deaton, A. (2004). Health in an Age of Globalization. Brookings Trade Forum. 2004.

Di Matteo, L. (2005). The macro determinants of health expenditure in the United States and Canada: assessing the impact of income, age distribution and time. Health Policy, 71(1). https://doi.org/10.1016/j. healthpol.2004.05.007

Farag, M., NandaKumar, A. K., Wallack, S., Hodgkin, D., Gaumer, G. y Erbil, C. (2012). The income elasticity of health care spending in developing and developed countries. International Journal of Health Care, Finance and Economics, 12, 145-162.

Fogel, R. W. (2009). Forecasting the cost of U.S. Health Care in 2040. Journal of Policy Modeling, 31(4), 482-488.

Forascepi Crespo, C. (2018). Chile: nuevos desafíos sanitarios e institucionales en un país en transición. Rev Panam Salud Publica, 42, e137. https://doi.org/10.26633/RPSP.2018. 137

Frenk, J., Frejka, T., Bobadilla, J. L., Stern, C., Lozano, R., Sepúlveda, J. y José, M. (1991) "La transición epidemiológica en América Latina. Boletín de la Oficina Sanitaria Panamericana (OSP),111(6).

Gerdtham, U. G. y Jonsson, B. (2000). International comparisons of health expenditure: theory, data and econometric analysis. In Handbook of Health Economics, Volume 1, Elsevier Science.

Getzen, T. E. (2000). Health care is an individual necessity and a national luxury: applying multilevel decision models to the analysis of health care expenditures. J. of Health Economics, 19, 259-270.

Gragnolati, M., Rofman, R., Apella, I., Troiano, S. (2014). Los años no vienen solos: oportunidades y desafíos economicos de la transicion demografica en Argentina. Washington, DC ; World Bank Group.

Hartwig, J. (2008). What drives health care expenditure? - Baumol's model of 'unbalanced growth' revisited. Journal of Health Economics, 27(3), 603-623

Hartwig, J. (2011). Can Baumol's model of unbalanced growth contribute to ex-plaining the secular rise in health care expenditure? An alternative test. Journal of Applied Economics, 43(2), 173-184.

Hausman, J. A. (1978). Specification Tests in Econometrics. Econometrica, 46(6), 1251-1271. https://doi. org/10.2307/1913827

Ho, C. Y. y Zhou, W. (2014). Unbalanced growth and health care expenditure. Economics of Transition, 22(4), 739-758.

Holly, A., Xu, K. y Saksena, P. (2011). The determinants of health expenditure: A Country-Level Panel Data Analysis. WHO Working Papers.

Kleiman, E. (1974). The Determinants of National Outlay on Health. In: Perlman M. (eds) The Economics of Health and Medical Care. International Economic Association Series. Palgrave Macmillan, London

Lis, M. (2016). Age or time-to-death — what drives health care expenditures? Panel data evidence from the OECD countries. IBS Working Papers 04/2016, Instytut Badan Strukturalnych. 
Maceira, D., Palacios, A., Urrutia, M. Espinola, N. y Nievas, M. (2017). Descentralización y estructura de las remuneraciones médicas en Argentina: un análisis comparado en cinco jurisdicciones. Revista Argentina Salud Pública, 8(30), 26-32.

Marino, A. et al. (2017). Future trends in health care expenditure: A modelling frame-work for cross-country forecasts”. OECD Health Working Papers, No. 95, OECD Publishing, Paris

Medeiros, J. y Schwierz, C. (2013). Estimating the drivers and projecting long-term public health expenditure in the European Union: Baumol's «cost disease» revisited. Belgium: Economic Papers 507.

Newhouse, J. P. (1977). Medical-Care Expenditure: A Cross-National Survey. The Journal of Human Resources, 12(1), 115-125.

Newhouse, J. P. (1992). Medical Care Costs: How Much Welfare Loss?. The journal of economic perspectives, 6, 3-21. 10.1257/jep.6.3.3.

OECD/Eurostat/WHO (2017). A System of Health Accounts 2011: Revised edition, OECD Publishing, Paris, https://doi.org/10.1787/9789264270985-en.

ONU (2015). Transformar nuestro mundo: la Agenda 2030 para el Desarrollo Sostenible (A/RES/70/)

OPS (2013). Cobertura Universal en Salud: Lecciones Internacionales Aprendidas y Elementos para su Consolidación en México. México, D.F. : OPS.

OPS (2017). Estado de salud de la población. https://www.paho.org/salud-en-las-americas-2017/ ?post_t_es=caracteristicas-de-la-poblacion-y-sus-tendencias\&lang=es

OPS (2018). Espacio fiscal para la salud en América Latina y el Caribe. Washington, D.C.: OPS; 2018

OPS (s/f). Transición demográfica en las Américas. http://hist.library.paho.org/Spanish/ EPID/18847.pdf

Parkin, D., McGuire, A. y Yule, B. (1987). Aggregate health care expenditures and national income. Is health care a luxury good?. Journal of Health Economics, 6(2), 109-127.

Rofman, R., Amarante Martinez, V. y Apella, I. (2016). Cambio demográfico y desafíos económicos y sociales en el Uruguay del siglo XXI. Washington, D.C. : World Bank Group.

Seshamani, M. y Gray, A. (2004). Ageing and health care expenditure: the red herring argument revisited. Health Economics, 13 (4), 303-314.

Solow, R. (1957). Technical Change and the Aggregate Production Function. The Review of Economics and Statistics, 39(3), 312-320.

Villalobos Dintrans, P. (2018). Is aging a problem?: Dependency, long-term care, and public policies in Chile. Rev Panam Salud Publica, 42, e168. https://doi.org/10.26633/ RPSP.2018.168 
WHO-WB (2014). Monitoring progress towards universal health coverage at country and global levels: framework, measures and targets. WHO https://apps.who.int/iris/handle/ 10665/112824

Xu, K., Soucat, A., Kutzin, J. et al. (2018). Public Spending on Health: A Closer Look at Global. Trends. Geneva: World Health Organization.

Yang, Z., Norton, E. C. y Stearns, S. C. (2003). Longevity and health care expenditures the real reasons older people spend more. Journals of Gerontology Series B: Psychological Sciences and Social Sciences, 58, 2-10

You, X. y Okunade, A. A. (2017). Income and Technology as Drivers of Australian Health Care Expenditure. Health Economics, 26(7), 853-862.

Zweifel, P., Felder, S. y Meiers, M. (1999). Ageing of population and health care expenditure: a red herring?. Health Economics, 8, 485-496. 